\title{
A Linearized Approach for the Seismic Response Analysis of Flexible Cable Net Structures
}

\author{
Yang XIANG, Yongfeng LUO, Xiaonong GUO*, Zhe XIONG, Zuyan SHEN \\ Department of Building Engineering, Tongji University \\ Shanghai 200092, P. R. China \\ * Corresponding author: guo-xiao-nong@tongji.edu.cn
}

\begin{abstract}
Cable net structures are characterized by significant geometrical nonlinear properties. The linear modal superposition method (LMSM), which ignores the effect of nonlinearity, is believed not suitable for the seismic response analysis of this kind of structures. To conquer the drawback of LMSM, a non-iterative linearization method (NILM) is proposed in this paper. An energy parameter named the "stiffness parameter" is utilized in generating the nonlinear equivalent single degree of freedom (ESDF) systems for the dominant vibration modes of cable nets. The nonlinear stiffness of an ESDF system is simulated by a quadratic function about the equivalent displacement. Based on a stochastic linearization process, the relationship between the equivalent linear stiffness and the displacement response of a modal ESDF system is established. Then the structural responses are computed according to the design response spectra through a non-iterative procedure. Finally, the effectiveness of the proposed NILM is illustrated by a numerical example that carried out on a trapezoid-shaped cable net supported curtain wall structure. It is demonstrated that the proposed NILM, which takes the stiffness hardening effect into consideration, could not only yield better results than the LMSM does, but also exhibit satisfying timesaving property over the nonlinear RHA.
\end{abstract}

Keywords: Cable net structure; nonlinear seismic response; equivalent single-degree-of-freedom system; equivalent linearization; non-iterative approach; seismic response spectrum; flexible curtain wall 


\begin{tabular}{|c|c|c|c|}
\hline \multicolumn{4}{|c|}{ Nomenclature } \\
\hline RHA & response history analysis & $a_{g}(t)$ & ground acceleration \\
\hline ESDF & equivalent single degree of free & $\Delta \chi_{n i}$ & load factor increment \\
\hline LDC & load-displacement curve & $k$ & stiffness \\
\hline SDF & single degree of free & $d$ & displacement \\
\hline$k^{*}$ & overall structural stiffness index & $a_{0}$ & coefficient in the k-d relationship \\
\hline$f_{u}$ & unit force & $b_{0}$ & coefficient in the k-d relationship \\
\hline$w_{u}$ & work done by the unit force & $F(d)$ & restoring force \\
\hline$d_{u}$ & displacement accordant to $f_{u}$ & $F_{e}(d)$ & linear restoring force \\
\hline$\delta_{u}$ & flexibility of the system & $k_{e}$ & equivalent stiffness \\
\hline$k_{u}$ & system stiffness corresponding to $w_{u}$ & SGP & stationary Gaussian process \\
\hline MDF & multi-degree of freedom & $d(t)$ & structural response process \\
\hline$\Delta \boldsymbol{P}_{i}$ & load increment vector in the $i^{\text {th }}$ load step & $\Delta F[d]$ & deviation process between $F(d)$ and $F_{e}(d)$ \\
\hline$\Delta v_{i}$ & displacement increment vector caused by $\Delta \boldsymbol{P}_{i}$ & $\mathrm{PDF}$ & probability density function \\
\hline$\Delta \boldsymbol{P}_{u i}$ & unitized vector corresponding to $\Delta \boldsymbol{P}_{i}$ & $p(d)$ & PDF of the $d(t)$ process \\
\hline$\Delta v_{u i}$ & displacement increment vector caused by $\Delta \boldsymbol{P}_{u i}$ & $\sigma_{d}$ & standard deviation of $d(t)$ \\
\hline$\left\|\Delta \boldsymbol{P}_{i}\right\|$ & norm of $\Delta \boldsymbol{P}_{i}$ & $a_{\text {resp }}$ & seismic acceleration response \\
\hline$\Delta w_{u i}$ & work done by $\Delta \boldsymbol{P}_{u i}$ & $T$ & vibrating period \\
\hline$k_{i}^{*}$ & equivalent structural stiffness of the $i^{\text {th }}$ load step & $d_{r e s p}$ & seismic displacement response \\
\hline$X_{n}$ & the $n^{\text {th }}$ modal displacement vector & $a_{\text {reap }} T(\omega)$ & transformed response spectrum \\
\hline$\chi_{n i}$ & static load factor of the $i^{\text {th }}$ load step & $a_{\text {reap }} T T$ & $m$ - $k$-based design response spectrum \\
\hline $\boldsymbol{F}_{n i}$ & force vector of the $i^{\text {th }}$ load step & NILM & non-iterative linearized method \\
\hline$S L F$ & static load factor & LMSM & linear modal superposition method \\
\hline$k_{n i}{ }^{*}$ & stiffness of the $n^{\text {th }}$ mode in the $i^{\text {th }}$ load step & A & cross-section area of cables \\
\hline$m_{n}{ }^{*}$ & equivalent mass of the $n^{\text {th }}$ modal ESDF system & E & Young-modulus \\
\hline$T_{n}$ & vibrating period of the $n^{\text {th }}$ mode & $\varepsilon$ & initial strain \\
\hline$\omega_{n}$ & circular frequency of the $n^{\text {th }}$ mode & $m$ & mass on the cable net façade model \\
\hline$\xi_{n}$ & damping ratio of the $n^{\text {th }}$ mode & $\xi_{n}$ & damping ratio of the $n^{\text {th }}$ mode \\
\hline$k_{n 0}{ }^{*}$ & initial equivalent stiffness of the $n^{\text {th }}$ ESDF system & $E M F$ & effective mass factor \\
\hline$F_{n i, j}$ & equivalent force on the $j^{\text {th }}$ node in the $i^{\text {th }}$ load step & $M P F$ & modal participate factor \\
\hline$X_{n, j}$ & the $j^{\text {th }}$ element of $\boldsymbol{X}_{\boldsymbol{n}}$ & PGA & peak ground acceleration \\
\hline$\alpha_{n i}$ & acceleration response of the $n^{\text {th }}$ mode & $k_{e}^{\mathrm{N}}$ & equivalent stiffness obtained by NILM \\
\hline$m_{j}$ & mass of the $j^{\text {th }}$ node & $k_{e}^{\mathrm{L}}$ & equivalent stiffness obtained by LMSM \\
\hline$\Gamma_{n}$ & modal participate factor of the $n^{\text {th }}$ mode & $d$ & overall structural displacement vector \\
\hline$F_{n i}{ }^{*}$ & equivalent seismic action on $m_{n}^{*}$ & $\varepsilon_{63}$ NILM & error of node 63 displacement of NILM \\
\hline & equivalent modal displacement & $\varepsilon_{F P} \mathrm{NILM}$ & error of cable force of NILM \\
\hline$\Delta F_{n i}{ }^{*}$ & force increment during the $i^{\text {th }}$ load step & $d_{63} \mathrm{NILM}^{2}$ & node 63 displacement computed by NILM \\
\hline$\Delta d_{n i}{ }^{*}$ & equivalent displacement increment & $d_{63}{ }^{\mathrm{RHA}}$ & node 63 displacement computed by RHA \\
\hline & equivalent displacement & $F_{p}^{\mathrm{NILM}}$ & cable force computed by NILM \\
\hline & displacement vector due to the $n^{\text {th }}$ mode & $F_{p}^{\mathrm{RHA}}$ & cable force computed by RHA \\
\hline & acceleration of the $n^{\text {th }}$ modal ESDF system & $\varepsilon_{63}$ LMSM & error of node 63 displacement of LMSM \\
\hline$v_{n}{ }^{*}$ & velocity of the $n^{\text {th }}$ modal ESDF system & $\varepsilon_{F p}$ LMSM & error of cable force of LMSM \\
\hline$F_{n}^{*}(d)$ & restoring force of the $n^{\text {th }}$ modal ESDF system & $d_{63}$ LMSM & node 63 displacement computed by LMSM \\
\hline$c_{n}$ & viscous damping coefficient of the $n^{\text {th }}$ mode & $F_{p}^{\text {LMSM }}$ & cable force computed by LMSM \\
\hline
\end{tabular}

\section{Introduction}

The monolayer cable net facades have been widely used in building structures over the last several decades. These flexible cable net structures mainly consist of pre-tensioned cables and glass panels, as shown in Fig.1. Because the structural stiffness and the bearing capacity are mainly provided by the cable members, the monolayer cable net systems are characterized by significant geometrical 
nonlinear behaviors. And this nonlinearity makes it pretty complicated to analyze the structural responses under dynamic excitations, such as seismic actions (Memari et al. [1]) and fluctuating wind pressures. To accurately compute these dynamic responses, a response history integration process is necessarily needed. Consequently, a series of response history analyses (RHA) is involved in either design or evaluation of a flexible cable net structure.

Fig.1. Cable net systems

In order to simplify the analysis procedure and, possibly, avoid the time consuming RHA process, notable works have been done on investigating the dynamic properties of the pre-tensioned cable net systems. Kwan [2] provided a simple technique for calculating the initial natural frequencies of cable nets, yet it was also pointed out that the period of a cable net is amplitude dependent. Vassilopoulou and Gantes [3,4] studied the natural frequencies and the corresponding vibration modes of saddle-formed cable networks with either rigid or flexible supports, and reported in details the nonlinear resonance phenomenon of this kind of structure. In investigating the behavior of cable net facades subjected to blast loads, the influence of negative phase loading were particularly discussed by Teich et al. [5]. During the last decade, Feng et al. [6,7,8,9] did a series of work on analyzing the dynamic responses of cable net structures. Firstly, Feng et al. [6] carried out static experiments and numerical simulations to study the working mechanism of monolayer cable net supported glass curtain walls. Besides, based on the continuous membrane theory, Feng et al. [7] established an equivalent single degree of freedom (ESDF) system and the corresponding nonlinear vibration governing equation of a cable net, and calculated its wind induced linear response by using a harmonic balance method. Meanwhile, Feng et al. [8] did dynamic model tests, as well as finite element analyses to learn the dynamic performance of cable net facades which considers the effects of the glass panels. Furthermore, according to the nonlinear ESDF system, Feng et al. [9] proposed the nonlinear response spectra for flexible cable net structures under four groups of site conditions. 
The research done by Wang et al, [10] Shi et al, [11] Wang and Zhao, [12], together with the aforementioned Feng et al.' work, $[6,7,8,9]$ all demonstrate that the role played by the nonlinear ESDF system is pretty essential in predicting the dynamic responses of the cable net systems. Yet all of the nonlinear ESDF systems in use are established based on a continuous theory, in which the deformed shape of a cable net facade is simulated by a sinusoidal curved surface and the boundary of the cable net model is assumed to be rectangular, moreover, the cable pretensions and nodal mass are considered as uniformly distributed. Taking the typical Feng et al.'s approach [7,9] for example, they assume the fundamental mode dominates the vibration of the cable net structure as shown in Fig.2, and express the deformation pattern of the overall system as a symmetric function:

$$
\varphi_{1}(x, z)=\sin \left(\pi\left(x / L_{x}\right)\right) \sin \left(\pi\left(z / L_{z}\right)\right)
$$

where $\varphi_{1}(x, z)$ is the out-of-plane displacement of the cable net at position $(x, z)$, while $x$ and $z$ are the horizontal and vertical location, respectively. Based on this continuous assumption, the vibration equation of the cable net could be derived.

Fig.2. Deformation pattern of the rectangular cable net in Feng's approach [9] For those cable net structures with irregular boundary shapes, the nonlinear ESDF systems, which represent the mechanical properties of the overall structures, can hardly be established according to the aforementioned approaches, as their two basic assumptions are not applicative anymore. One of them is the sinusoidal curved deformation assumption, and the other is the uniform distribution of pretension and mass assumption. For instance, in designing of the cable net curtain wall of the Beijing New Poly Plaza, (Yang et al. [13]) the structural dynamic properties and responses can only be obtained by a thoroughly executed RHA process, just because the elevation of the curtain wall is "L-shaped".

Besides of the geometrical topology limitation exists in the boundary shapes of cable net structures, another shortcoming of the aforementioned nonlinear approaches is that the amplitude dependent 
nonlinear stiffness of the ESDF system is not satisfyingly calculated. Feng et al. [14] proposed an applicative approach for nonlinear wind-resistance design of cable net systems, which could generate the equivalent linear stiffness of the nonlinear ESDF system through a non-iterative process by considering the dynamic input characteristic and the structural response. While for cable nets bearing severe seismic actions, Feng et al.'s approach is limited, because that the fluctuating wind pressures always act on the deformed positions of cable nets where the systems balance the average wind pressures, consequently, nonsymmetrical structural stiffness properties would appear as the system vibrates around this average-pressure-balanced positions.

Focusing on the two problems mentioned above, this paper developed a "stiffness-parameter-based" procedure to establish the nonlinear ESDF systems for irregular shaped cable net structures. Besides of the fundamental vibration mode, higher-order modes are taken into consideration in calculating the seismic responses of cable nets. Moreover, the equivalent linear stiffness of the nonlinear ESDF systems is determined directly based on the design seismic response spectra without involving iterative computations. The overall process of the proposed non-iterative method for the seismic response analysis of flexible cable nets is listed in details for practical applications. And finally, the effectiveness of the proposed method is illustrated by a numerical example that carried out on a trapezoid-shaped cable net supported curtain wall structure.

\section{Structural stiffness of a nonlinear system and the establishment of the ESDF system}

Generally, engineers use the load-displacement curves (LDC) to get information about the structural stiffness properties. These curves show precise stiffness evolution processes for regular buildings. But for irregular structures, especially those involve geometrical nonlinear effects, the LDCs are believed no longer suitable for describing the structural stiffness. This is mainly caused by the following two reasons. Firstly, it can hardly select the key node for an irregular structure as specifically as that of a regular one. Here the key node refers to the node whose LDC is preferably used to demonstrate the overall structural stiffness property. Secondly, due to the geometrical 
nonlinear effects, the LDCs given by different nodes could exhibit obvious mutual deviation. In view of these two drawbacks, an applicative index $k^{*}$ is established to numerically describe the structural nonlinearity instead of the LDCs in this section. The basic concept and the establishing process of the index $k^{*}$ is given hereby.

\subsection{The overall structural stiffness index $k^{*}$}

For a single degree of freedom (SDF) system bearing a unit force $f_{u}$, the work done by $f_{u}$ is numerically equal to half of the displacement occurred in the force direction, as shown by equation (2):

$$
w_{u}=\frac{1}{2} \cdot f_{u} \cdot d_{u}=\frac{1}{2} \cdot\left\|d_{u}\right\|
$$

where $w_{u}$ and $d_{u}$ are the work and the displacement in accordance with $f_{u}$, respectively.

The flexibility of the system, $\delta_{u}$, is equal to the displacement $d_{u}$ herein, and the system stiffness $k_{u}$ would be calculated as:

$$
k_{u}=\frac{1}{\delta_{u}}=\frac{1}{\left\|d_{u}\right\|}=\frac{1}{2 \cdot w_{u}}
$$

By extending the stiffness concept for SDF systems to multi-degree of freedom (MDF) systems, the overall stiffness of a geometrically nonlinear structure could also be appropriately measured.

For the $i^{\text {th }}$ load step of a static nonlinear analysis process, the load increment vector and the corresponding displacement increment vector could be expressed as $\Delta \boldsymbol{P}_{\boldsymbol{i}}$ and $\Delta \boldsymbol{v}_{\boldsymbol{i}}$, respectively. Then the unit force vector $\Delta \boldsymbol{P}_{u i}$, as well as the displacement vector $\Delta \boldsymbol{v}_{u i}$ caused by $\Delta \boldsymbol{P}_{u i}$, can be computed according to equation (4) and (5).

$$
\begin{aligned}
\Delta \boldsymbol{P}_{u i} & =\frac{\Delta \boldsymbol{P}_{i}}{\left\|\Delta \boldsymbol{P}_{i}\right\|} \\
\Delta \boldsymbol{v}_{u i} & =\frac{\Delta \boldsymbol{v}_{i}}{\left\|\Delta \boldsymbol{P}_{i}\right\|}
\end{aligned}
$$

where $\left\|\Delta \boldsymbol{P}_{\boldsymbol{i}}\right\|$ is the norm of the vector $\Delta \boldsymbol{P}_{\boldsymbol{i}}$. Then we can compute the work done by $\Delta \boldsymbol{P}_{\boldsymbol{u} i}$ as the following equation shows: 


$$
\Delta w_{u i}=\frac{1}{2} \cdot \Delta \boldsymbol{P}_{u i}{ }^{T} \cdot \Delta \boldsymbol{v}_{u i}
$$

where $\Delta \boldsymbol{P}_{u i}{ }^{\boldsymbol{T}}$ is the transpose of $\Delta \boldsymbol{P}_{\boldsymbol{u} i}$.

Accordingly, the equivalent structural stiffness of the $i^{\text {th }}$ load step could be obtained through equation (7).

$$
k_{i}^{*}=\frac{1}{2 \cdot \Delta w_{u i}}=\frac{1}{\Delta \boldsymbol{P}_{u i}{ }^{T} \cdot \Delta \boldsymbol{v}_{u i}}=\frac{\left\|\Delta \boldsymbol{P}_{i}\right\|^{2}}{\Delta \boldsymbol{P}_{i}^{T} \cdot \Delta \boldsymbol{v}_{i}}
$$

As can be seen from the generation process of $k^{*}$ given above, the value of $k^{*}$ is determined by all of the structural nodal load-displacement relationships. Thus, $k^{*}$ is a parameter that could define the overall stiffness of an irregular nonlinear structure. During a static loading process, the change of the stiffness could be observed through the gradual variation of $k^{*}$ of each load step.

\subsection{The ESDF systems for dynamic analysis of cable net structures}

For those flexible cable nets with irregular geometries, the dynamic responses of higher-order modes need to be considered in some cases. Therefore, the modal ESDF systems for all of the involved vibration modes ought to be established correspondingly. Detailed establishing processes of the nonlinear ESDF systems are provided in the following 1 5 steps.

1. For each dominant vibration mode, a nonlinear static analysis is carried out using the mode shape proportioned load pattern, as given in the equation

$$
\boldsymbol{F}_{n i}=\chi_{n i} \cdot \boldsymbol{X}_{n}
$$

where $\boldsymbol{X}_{\boldsymbol{n}}$ is the $n^{\text {th }}$ mode displacement vector, $\chi_{n i}$ and $\boldsymbol{F}_{\boldsymbol{n} i}$ are the static load factor $(S L F)$ and the force vector of the $i^{\text {th }}$ load step, respectively.

According to equation (4), (5), (6) and (7), the stiffness parameter of each load step, $k_{n i}{ }^{*}$, could be computed using the data obtained in the static analysis procedure, and an approximate relationship between $\chi_{n i}$ and $k_{n i}{ }^{*}$ could be derived simultaneously. For cable net systems, a quadratic function with fitted coefficients could describe the $\chi_{n i}-k_{n i}{ }^{*}$ relationship accurately.

2. In order to generate the equivalent mass $m_{n}{ }^{*}$ of the $n^{\text {th }}$ modal ESDF system, the following 
equations are used:

$$
\begin{gathered}
\omega_{n}=\frac{2 \pi}{T_{n}} \\
\omega_{n}=\sqrt{\frac{k_{n 0}^{*}}{m_{n}^{*}}} \cdot \sqrt{1-\xi_{n}^{2}}
\end{gathered}
$$

where $T_{n}, \omega_{n}$ and $\xi_{n}$ are the period, the circular frequency and the damping ratio of the $n^{\text {th }}$ mode, respectively, and $k_{n 0}{ }^{*}$ is the initial stiffness of the nonlinear system which can be calculated by setting $\chi_{n i}=0$ in the $\chi_{n i}-k_{n i}{ }^{*}$ relationship.

Substituting equation (9) into equation (10), after a simple transformation we obtain equation (11).

$$
m_{n}^{*}=\frac{1}{4 \pi^{2}} \cdot k_{n 0}^{*} \cdot T_{n}^{2} \cdot\left(1-\xi_{n}^{2}\right)
$$

For a structure with low damping ratio, equation (10) and (11) can be simplified, as equations (12) and (13) shows.

$$
\begin{gathered}
\omega_{n}=\sqrt{\frac{k_{n 0}{ }^{*}}{m_{n}^{*}}} \\
m_{n}{ }^{*}=\frac{1}{4 \pi^{2}} \cdot k_{n 0}{ }^{*} \cdot T_{n}^{2}
\end{gathered}
$$

3. Since the force vector $\boldsymbol{F}_{\boldsymbol{n}}$ given by equation (8) can be regarded as the equivalent seismic action of the corresponding mode, $\boldsymbol{F}_{\boldsymbol{n} i}$ could be rewritten into the following format:

$$
F_{n i, j}=\chi_{n i} \cdot X_{n, j}=\alpha_{n i} \cdot m_{j} \cdot \Gamma_{n} \cdot X_{n, j}
$$

where $F_{n i, j}$ is the equivalent force acts on the $j^{\text {th }}$ node in the $i^{\text {th }}$ load step of the static nonlinear analysis of the $n^{\text {th }}$ mode, $X_{n, j}$ is the $j^{\text {th }}$ element of $\boldsymbol{X}_{n}, \alpha_{n i}$ is the acceleration response of the $n^{\text {th }}$ mode, $m_{j}$ is the mass of the $j^{\text {th }}$ node, and $\Gamma_{n}$ the modal participate factor. In fact, the right part of equation (14) expresses the equivalent static forces associated with the $n^{\text {th }}$ mode response. (Chopra [15]) In converting the MDF formula into the ESDF formula, we can replace the $m_{j}$ with the equivalent mass $m_{n}{ }^{*}$ in equation (14), and thus the ESDF format of this equation could be derived, as the following equation shows: 


$$
F_{n i}^{*}=\chi_{n i} \cdot X_{n}^{*}=\alpha_{n i} \cdot m_{n}^{*} \cdot \Gamma_{n} \cdot X_{n}^{*}
$$

where $F_{n i}{ }^{*}$ and $X_{n}{ }^{*}$ are the equivalent seismic action on $m_{n}{ }^{*}$ and the equivalent modal displacement assumed, respectively.

Indicated by equation (15), $\alpha_{n i}$ could be computed in the further step,

$$
\alpha_{n i}=\frac{\chi_{n i}}{m_{n}^{*} \cdot \Gamma_{n}}
$$

and the equivalent force $F_{n i}{ }^{*}$ of the ESDF system in accordance with the $i^{\text {th }}$ load step and the $n^{\text {th }}$ mode could be obtained by using equation (17).

$$
F_{n i}^{*}=\alpha_{n i} \cdot m_{n}^{*}
$$

4. The equivalent displacement increment $\Delta d_{n i}{ }^{*}$ of the $n^{\text {th }}$ modal ESDF system due to the $i^{\text {th }}$ load step can be calculated by equation (18):

$$
\Delta d_{n i}^{*}=\frac{\Delta F_{n i}^{*}}{k_{n i}^{*}}
$$

where $\Delta F_{n i}{ }^{*}$ is the force increment during the $i^{\text {th }}$ load step. Based on the $\Delta d_{n i}{ }^{*}$ obtained in each load step, we can draw the load-displacement curve, that is, the $F_{n i}{ }^{*}-d_{n i}{ }^{*}$ relationship for the ESDF system of the $n^{\text {th }}$ mode.

5. Combining equation (16) and (17), we can get the relationship between $\chi_{n i}$ and $F_{n i}{ }^{*}$, just as equation (19) shows.

$$
F_{n i}^{*}=\frac{\chi_{n i}}{\Gamma_{n}}
$$

Based on the above equation, we can convert the former obtained $\chi_{n i}-k_{n i}{ }^{*}$ function into the $F_{n i}-k_{n i}{ }^{*}$ format. Then a $k_{n i}{ }^{*}-d_{n i}{ }^{*}$ relationship could be reached as the relationships of $F_{n i}{ }^{*}-d_{n i}{ }^{*}$ and $F_{n i}-k_{n i}{ }^{*}$ being considered together. For a cable net system, the $k_{n i}{ }^{*}-d_{n i}{ }^{*}$ relationship expressed in terms of $k_{n}{ }^{*}=f\left(d_{n}{ }^{*}\right)$, would also exhibit a quadratic property as $\chi_{n i}-k_{n i}{ }^{*}$ does.

The equation of motion for the $n^{\text {th }}$ modal ESDF system could be written as follow:

$$
m_{n}^{*} \cdot a_{n}^{*}+c_{n} \cdot v_{n}^{*}+F_{n}^{*}(d)=-m_{n}^{*} \cdot a_{g}(t)
$$


where $a_{n}{ }^{*}, v_{n}{ }^{*}$ and $F_{n}{ }^{*}(d)$ are the acceleration, the velocity and the restoring force of the system, respectively, $c_{n}$ is the viscous damping coefficient, and $a_{g}(t)$ the ground acceleration of the earthquake excitation.

It can be seen from the step 1 that the force increment vector $\Delta \boldsymbol{F}_{\boldsymbol{n} i}$ of the $i^{\text {th }}$ load step is governed by the load factor increment $\Delta \chi_{n i}$ only, indicating that the mode shapes do not vary as the deformation of the structure increases. Although this fixed-mode-shape assumption is not valid theoretically for geometrical nonlinear structures, it is believed that the neglecting of the tiny variations of the vibration modes will not bring forward unacceptable errors.

\section{Stochastic linearization approach of the geometrical nonlinear system}

In general, the relationship between the stiffness $k$ and the displacement $d$ could be considered as a quadratic function, either for a single cable or for a cable net. The $k-d$ function is as follow:

$$
k=f(d)=a_{0} \cdot d^{2}+b_{0}
$$

where $a_{0}$ and $b_{0}$ are coefficients which could be determined by a polynomial fitting approach using the $k_{n i}{ }^{*}-d_{n i}{ }^{*}$ data obtained in the nonlinear static analysis.

According to the $k$ - $d$ function given by equation (21), the relationship between the restoring force $F(d)$ and the displacement $d$ could be derived after integral, as shown by equation (22):

$$
F(d)=\frac{1}{3} \cdot a_{0} \cdot d^{3}+b_{0} \cdot d+c_{0}
$$

where $c_{0}$ is an integral constant. It can be easily conducted that $c_{0}=0$ because $F(d)$ ought to be zero when the displacement $d$ is equal to zero. Hence, the standard $F(d)$ formula is as equation (23) shows.

$$
F(d)=\frac{1}{3} \cdot a_{0} \cdot d^{3}+b_{0} \cdot d
$$

Substituting equation (23) into equation (20), the governing equation of the nonlinear dynamic response of the $n^{\text {th }}$ modal ESDF system could be reached, as shown in equation (24).

$$
m_{n}^{*} \cdot \ddot{d}_{n}^{*}+c_{n} \cdot \dot{d}_{n}^{*}+b_{0} \cdot d+\frac{a_{0}}{3} \cdot d^{3}=-m_{n}^{*} \cdot a_{g}(t)
$$


To accurately solve this dynamic equation, a nonlinear RHA process is needed. Besides of the enormous input and output evolved, another shortcoming of the RHA is that the results are overly dependent on the input seismic excitation records. To avoid these shortcomings, seismic response spectra are recommended by design codes and guideline documents. Seeing that the design response spectra are set for linear situations, a linearization approach for dealing with the nonlinear dynamic problem governed by equation (24) is developed hereby.

The standard way of carrying out a linearization process is to minimize the mean square measure of the deviation between the structural responses given by the nonlinear system and the linearized system (Naess [16]; Lin and Miranda [17]). For the modal ESDF systems of a cable net discussed herein, the equivalent restoring force $F$ is studied to generate the linearization process.

Replacing the nonlinear restoring force $F(d)$ with $F_{e}(d)$, which is linearly related to the displacement $d$ as shown in equation (25), the goal of the linearization procedure becomes to find the reasonable value of $k_{e}$, correspondingly.

$$
F_{e}(d)=k_{e} \cdot d
$$

In finding $k_{e}$, the deviation between $F(d)$ and $F_{e}(d)$ is investigated. We can firstly assume that the seismic ground motion process $a_{g}(t)$ is a stationary Gaussian process (SGP) with a zero mean value, and further predict the structural response process $d(t)$ to be a zero mean valued SGP as well. Therefore, the deviation process emerging between $F(d)$ and $F_{e}(d)$ could be expressed as follow:

$$
\Delta F[d(t)]=F[d(t)]-F_{e}[d(t)]
$$

where $F[d(t)]$ and $F_{e}[d(t)]$ are the stochastic process of the nonlinear restoring force and the equivalent linear restoring force, respectively. In the following context, $F[d(t)]$ and $F_{e}[d(t)]$ will be donated as $F(d)$ and $F_{e}(d)$ for short. Thus the mean square value of the deviation process $\Delta F[d]$ is

$$
E\{[\Delta F(d)]\}^{2}=\int_{-\infty}^{\infty}\left[F(d)-F_{e}(d)\right]^{2} \cdot p(d) \mathrm{d} d
$$

where $p(d)$ is the probability density function (PDF) of the response process $d(t)$. As $d(t)$ has been assumed to be a zero mean valued SGP, its PDF is 


$$
p(d)=\frac{1}{\sqrt{2 \pi} \sigma_{d}} \exp \left(-\frac{d^{2}}{2 \sigma_{d}^{2}}\right)
$$

where $\sigma_{d}$ is the standard deviation of $d(t)$.

The best value of $k_{e}$ would give the minimum $E\{[\Delta F(d)]\}^{2}$, which indicates the minimum deviation between $F(d)$ and $F_{e}(d)$. So it is required that the $E\{[\Delta F(d)]\}^{2}$ reaches a stationary value when $k_{e}$ is satisfying. Therefore, we can reach equation (29) for figuring out the value of $k_{e}$.

$$
\frac{\partial E\{[\Delta F(d)]\}^{2}}{\partial k_{e}}=0
$$

Substituting equation (29) into equation (27), we get equation (30).

$$
-2 \int_{-\infty}^{\infty}\left[F(d)-F_{e}(d)\right] \cdot \frac{\partial F_{e}(d)}{\partial k_{e}} \cdot p(d) \mathrm{d} d=0
$$

Then by substituting equation (23) and (25) into equation (30), we can further obtain a formula as shown in the following equation (31).

$$
-\frac{2}{3} \cdot a_{0} \int_{-\infty}^{\infty} d^{4} \cdot p(d) \mathrm{d} d-2 \cdot\left(b_{0}-k_{e}\right) \int_{-\infty}^{\infty} \mathrm{d}^{2} \cdot p(d) \mathrm{d} d=0
$$

The first and the second integral term in the above equation are the fourth-order central moment and the second-order central moment of the $d(t)$ process, respectively. These central moments could be calculated according to equation (32) and (33).

$$
\begin{gathered}
\int_{-\infty}^{\infty} d^{4} \cdot p(d) \mathrm{d} d=E\left(d^{4}\right)=\sigma_{d}^{4} \cdot(4-1) ! !=3 \cdot \sigma_{d}{ }^{4} \\
\int_{-\infty}^{\infty} \mathrm{d}^{2} \cdot p(d) \mathrm{d} d=E\left(d^{2}\right)=\sigma_{d}{ }^{2}
\end{gathered}
$$

Substituting the former two equations into equation (31), after rearrangement we obtain

$$
k_{e}=a_{0} \cdot \sigma_{d}^{2}+b_{0}
$$

which expresses the relationship between $k_{e}$ and the standard deviation of the $d(t)$ process.

For a zero mean valued stochastic $d(t)$ process generated by an earthquake excitation, its standard deviation is numerically equal to the displacement response $d$, hence equation (34) could be rewritten as equation (35), which illustrates the relationship between $k_{e}$ and $d$ in a more direct manner. 


$$
k_{e}=a_{0} \cdot d^{2}+b_{0}
$$

The above correlation between $k_{e}$ and $d$ means that the system is geometrically hardening. In finding out $k_{e}$, the value of $d$ ought to be determined firstly. This correlation leads to an iterative process in obtaining the optimal pair of $k_{e}$ and $d$, in which the computed values are modified during each iterative step until they are within an allowable tolerance to the assumed values. To avoid the iterative, a direct way for calculating $k_{e}$ and $d$ is developed based on the linear design response spectra provided by design codes and documents.

\section{Non-iterative process for computing the displacement responses of the ESDF systems}

The design acceleration response spectra are commonly given in an $a_{r e s p}-T$ format, herein $a_{\text {resp }}$ and $T$ denote the seismic acceleration response and the vibrating period of the modal ESDF system, respectively. According to the $a_{\text {resp }}$, the displacement response $d_{\text {resp }}$ could be conducted through equation (36) (Chopra [15])

$$
d_{\text {resp }}(T)=\frac{T^{2}}{4 \pi^{2}} \cdot a_{\text {resp }}(T)
$$

By application of equation (9), the $T$-based $d_{\text {resp }}-a_{\text {resp }}$ relationship could be transformed into the following one in which the variable $T$ is replaced by the $\omega$ :

$$
d_{r e s p}(\omega)=\frac{1}{\omega^{2}} \cdot a_{r e s p}^{T}(\omega)
$$

where $a_{\text {reap }}^{T}(\omega)$ is the transformed response spectrum expression which uses $\omega$ as the basic variable. In further steps, equation (10) is used for rewriting the $\omega$-based $d_{\text {resp }}-a_{\text {resp }}$ relationship into the following form

$$
d_{\text {resp }}(k)=\frac{m}{k} \cdot a_{\text {resp }}{ }^{T T}(m, k)
$$

where $a_{\text {reap }}^{T T}(m, \omega)$ is the $m$ - $k$-based version of the design response spectrum, deviated from the $a_{\text {reap }}{ }^{T}(\omega)$ expression, and $d_{\text {reap }}(k)$ is the displacement response which can be determined by the stiffness $k$ when the value of $m$ is preset. For systems with a low damping ratio, equation (12) could be used instead of equation (10) in conducting this transforming process. 
To illustrate the transforming process, three acceleration response spectra recommended by design codes or documents are transformed. (ASCE [18]; EN1998-1 [19]; MOHURD and AQSIQ of China [20]) The initial $a_{r e s p}-T$ format of these spectra and their corresponding $m-k-d$ format expressions are given in the appendix of this paper.

Back to the problem of computing $k_{e}$ and $d$, another equation between $k_{e}$ and $d$, besides of equation (35), could be obtained by substituting the $m_{n}{ }^{*}$ into the $m-k-d$ format design response spectra, as given by equation (39).

$$
d\left(k_{\mathrm{e}}\right)=\frac{m_{n}^{*}}{k_{e}} \cdot a_{r e s p}{ }^{T T}\left(m_{n}^{*}, k_{e}\right)
$$

By solving the simultaneous equations consist of equation (35) and equation (39), the values of $d$ and $k_{e}$ are obtained.

\section{The complete process of the proposed calculation approach}

According to the value of $d$, the structural displacement vector $\boldsymbol{d}_{\boldsymbol{n}}$ due to each dominant mode could be extracted from the static analysis database. Combining the modal displacement vectors with either the SRSS rule or the CQC rule, the displacement of a cable net system could finally be obtained.

To summarize the proposed non-iterative linearized method (NILM) for seismic response analysis of the flexible cable net structures, a flow chart is given in Fig.3. In the flow chart, the procedures for carrying out a NILM are listed step by step.

Fig.3. The non-iterative linearized analysis procedure

Comparing the NILM to the linear modal superposition method (LMSM), two extra steps, including the nonlinear static analyses and the polynomial fittings, are needed by the former. But the computing cost brought forward by these extra steps is negligible. Meanwhile, the advantages exhibited by the NILM are obvious. On one hand, comparing to the LMSM, the NILM takes the nonlinear characteristics of the structures into consideration, on the other hand, comparing to the RHA method, the design response spectra are used and much computing consumption is avoided. 


\section{Numerical Example}

\subsection{The cable net supported curtain wall model}

To illustrate the effectiveness of the proposed NILM, a numerical example, carried out on a right-angled trapezoidal cable net supported curtain wall structure, is given in this section. The elevation of the curtain wall, as well as its dimensions, is shown in Fig.4. The cable net system consists of 11 horizontal cables and 14 vertical ones. All cables are supposed to have the same cross-section with area $A=150 \mathrm{~mm}^{2}$, and their material is assumed linearly elastic with Young-modulus $E=2.06 \mathrm{GPa}$. The initial strain of each cable is set to $\varepsilon=0.0045$, which provides the curtain wall system with a reasonable initial stiffness. The ends of the cables are pinned to the frames on the edges. The edge frames are assumed rigid, making all the pinned cable ends fixed. A lumped mass model, with an equivalent mass $m=60 \mathrm{~kg}$ at each intersection of the cables, are set to the system. The damping ratio of each vibration mode is $\xi_{n}=0.02$. The finite element model (FEM) is established in the ANSYS program, the cables and the masses are simulated by the link180 element and the mass 21 element, respectively. The node numbers of the FEM of the cable net are shown in Fig.5.

Fig.4. Elevation of the trapezoidal cable net supported curtain wall system

Fig.5. Node numbers of the FEM of the cable net

\subsection{Dominant modes and ESDF systems}

The cable pretension process is firstly simulated, and the modal analysis of the system is executed on the pre-stressed status. According to the modal analysis results, the $1^{\text {st }}$, the $3^{\text {rd }}$ and the $8^{\text {th }}$ eigen-modes have the largest effective mass factors $(E M F)$ and the modal participate factors $(M P F)$, therefore, these three modes are selected as the dominant modes of the curtain wall structure. The shapes of the dominant modes are drawn in Fig.6. As can be seen in the figure, the $1^{\text {st }}$ and the $8^{\text {th }}$ modes are approximately symmetric while the $3^{\text {rd }}$ is approximately anti-symmetric. 
Fig.6. Dominant vibration modes of the cable net supported curtain wall model

By carrying out a series of static nonlinear analyses, using the modal shape proportioned load patterns, the equivalent stiffness $k_{n i}{ }^{*}$ of each load step is computed according to equation (7) for each dominant mode. The equivalent displacement $\Delta d_{n i}{ }^{*}$ occurred during each load step is obtained by using equation (18) simultaneously. And thereby, the relationships between $\chi_{n}$ and $k_{n}^{*}$ of the modal ESDF systems, as well as the equivalent $\chi_{n}-d_{n}{ }^{*}$ curves are obtained, as Fig.7 and Fig.8 show.

As can be seen from Fig.7, the relationship between $\chi_{n}$ and $k_{n}{ }^{*}$ for each mode exhibits quite similar characteristic as a quadratic parabola does. These relationship curves are all symmetric about the line $\chi=0$, indicating that the degrees of hardening in the two out-of-plane directions are the same as long as the absolute values of displacements in the two directions are equal. Correspondingly, the relationship between $\chi_{n}$ and $d_{n}{ }^{*}$ are approximate cubic polynomials, as Fig.8 demonstrates. The nonlinear effect of the $1^{\text {st }}$ modal ESDF system is pretty obvious, while this effect is not that clear for the $3^{\text {rd }}$ and the $8^{\text {th }}$.

According to the $\chi_{n}$ and $k_{n}{ }^{*}$ data, the relationships between $F_{n}{ }^{*}$ and $k_{n}{ }^{*}$ are generated. Using the least square approach, the values of $a_{0}$ and $b_{0}$ in equation (21) are gained based on the $F_{n}{ }^{*}$ and $k_{n}{ }^{*}$ data. Meanwhile, the initial elastic stiffness $k_{n 0}{ }^{*}$ for each modal ESDF system is set equal to the value of $b_{0}$. The equivalent ESDF masses are also computed as equation (13) indicates.

For the three dominant modes of the cable net model, the values of their vibration period $T$, the modal EMF and the MPF, the $k_{n}{ }^{*}$ and $m_{n}{ }^{*}$ of the ESDF systems, as well as the coefficients $a_{0}$ and $b_{0}$, are listed in Table 1.

Table 1 Information of the dominant vibration modes and the corresponding ESDF systems

Fig.7. Relationship between $\chi_{n}$ and $k_{n}^{*}$ 
Fig.8. The equivalent load-displacement curves of the dominant vibration modes

\subsection{Earthquake excitations}

Sixteen earthquake excitation records were selected for calculating the seismic response of the cable net model. Detailed information of these earthquake records are listed in Table 2. Their acceleration response spectra are plotted in Fig.9.a. For each earthquake record, the acceleration response spectrum is calculated for a set of 200 periods of vibration between 0.02 to $4.00 \mathrm{~s}$ with an increment of 0.02 s. The displacement response spectra, plotted in Fig.9.b, are calculated based on equation (36).

Table 2 List of the earthquake excitations

Fig.9. Linear response spectra of the seismic waves

\subsection{Node displacement prediction}

All of the earthquake records are amplified to nine degrees of intensity with the PGA of $0.3 \mathrm{~g}, 0.4 \mathrm{~g}$, $0.5 \mathrm{~g}, 0.6 \mathrm{~g}, 0.7 \mathrm{~g}, 0.8 \mathrm{~g}, 0.9 \mathrm{~g}, 1.0 \mathrm{~g}$, and $1.1 \mathrm{~g}$, respectively. Herein $\mathrm{g}$ indicates the gravity with the value of $9.8 \mathrm{~m} / \mathrm{s}^{2}$. Based on the $a_{r e s p}-T$ format response spectra of these earthquake records with different PGAs, the $m-k-d$ format spectra are obtained through the aforementioned spectrum transforming procedure.

In contrast to the design spectra, there are no functional expressions for the spectra given by the earthquake records in this numerical example. Therefore, instead of solving the simultaneous equations consist of equation (35) and (39), a graphic approach is adopted in computing the optimal pair of $k_{e}$ and $d$. For each dominant modal ESDF system, substituting the value of $m_{n}{ }^{*}$ into the $m-k-d$ format spectra, a set of $k-d$ curves are gained. By plotting a spectrum-based $k-d$ curve and the quadratic parabola relationship curve of $k_{e^{-}} d$ in the same coordinate system, the intersection of the 
two curves would provide the wanted value of $k_{e}$ and $d$.

Fig.10 Fig.12 show the status of the intersections provided by the aforementioned curves for the three dominant modes. In order to provide accurate locations of the intersection points of the $k_{e^{-}} d$ lines and the response spectra lines for this numerical example, the response spectra plotted in Fig.10 Fig.12 are computed for a set of 400 periods of vibration between 0.1 to $0.4 \mathrm{~s}$ with an increment of $0.001 \mathrm{~s}$. For each modal ESDF system, the value of $d$ given by the spectrum-based $k$ - $d$ curve gets lager as the PGA of the seismic input increases. For the purpose of comparison, the linear $k$ - $d$ relationship which used by the LMSM is also plotted in each chart in Fig.10 Fig.12. Differs from the nonlinear $k_{e^{-}} d$ curves, the linear $k-d$ curves are upright straight lines which mean that the value of $k$ does not vary no matter how the value of $d$ changes.

Fig.10. Graphic solutions for the ESDF responses of the $1^{\text {st }}$ mode

Fig.11. Graphic solutions for the ESDF responses of the $3^{\text {rd }}$ mode

Fig.12. Graphic solutions for the ESDF responses of the $8^{\text {th }}$ mode

Let $k_{e}^{\mathrm{N}}$ be the equivalent stiffness $k_{e}$ acquired according to the nonlinear $k$ - $d$ relationship, and $k_{e}^{\mathrm{L}}$ be the linear stiffness that demonstrated by the upright lines in the charts. Focusing on the chart of the $1^{\text {st }}$ mode, it is thus clear that the value of $k_{e}^{\mathrm{L}}$ is much less than that of $k_{e}{ }^{\mathrm{N}}$, regardless of the earthquake record. Meanwhile, it can be seen that the larger the PGA is, the more deviation between $k_{e}{ }^{\mathrm{L}}$ and $k_{e}^{\mathrm{N}}$ shows. Besides of $k_{e}$, the corresponding displacements $d$ also exhibit mutual differences between the linear results $d^{\mathrm{L}}$ and the nonlinear results $d^{\mathrm{N}}$ for the $1^{\text {st }}$ vibration mode. Generally, the value of $d^{\mathrm{N}}$ is smaller than that of $d^{\mathrm{L}}$. While for the $3^{\text {rd }}$ and the $8^{\text {th }}$ mode, the deviation between the linear and nonlinear solutions is not that obvious like the $1^{\text {st }}$ mode does. So it can be inferred that the 
nonlinear effects of the $3^{\text {rd }}$ and the $8^{\text {th }}$ modes are negligible.

Based on the statistical results of the optimal pairs of $k_{e}$ and $d$, the corresponding $S L F$ s $\chi$ could be drawn from the data base of the nonlinear static analysis process. The numerical values of the $S L F$ s for both linear and nonlinear situations for the $1^{\text {st }}$ mode are provided in Table 3 . For the $3^{\text {rd }}$ and the $8^{\text {th }}$ mode, seeing that the deviations between the linear and nonlinear values are tiny, only the linear SLFs are listed in Table 4 and Table 5 for these two modes, respectively.

According to the $S L F \mathrm{~s}$, the global displacement of each dominant mode $\boldsymbol{d}_{\boldsymbol{n}}$ is extracted. Then the overall displacement $\boldsymbol{d}$ of the cable net structure is computed by following the SRSS rule as shown in equation (40).

$$
\boldsymbol{d}=\sqrt{\sum \boldsymbol{d}_{n}^{2}}
$$

Table $3 S L F$ s of the $1^{\text {st }}$ mode corresponding to the ESDF system displacements

Table 4 Linear $S L F$ s of the $3^{\text {rd }}$ and the $8^{\text {th }}$ mode corresponding to the ESDF system displacements

\subsection{Accuracy of the proposed NILM}

The nodal displacements in the out-of-plane direction calculated by the RHA approach, the LMSM and the proposed NILM are drawn in Fig.13 for the $B c_{-} 310$ earthquake excitation with the $\mathrm{PGA}=1.1 \mathrm{~g}$, respectively. The nodal displacement values of the RHA approach plotted in Fig.13 were recorded at the time when the central node (node 63) of the cable net reaches its peak absolute value during the response history. It can be seen from Fig.13 that the deformation of the cable net is mainly governed by its fundamental vibration mode. The peak displacement value is given by node 63 , which locates at the middle span of the cable net. The deformed shape provided by either the LMSM or the NILM is quite alike to the benchmark result given by the RHA approach. Generally, the model deforming patterns calculated by the three approaches, including the RHA method, the LMSM and 
the NILM, show good accordant degrees for every seismic input situation. The main differences between the LMSM and the NILM regarding to the computing accuracies, will be examined by indexes given in the following context.

Fig.13. Nodal displacements obtained under the $B c_{-} 310$ earthquake excitation

Firstly, to investigate the accuracy of the NILM relative to the RHA method, two variables $\varepsilon_{63}{ }^{\text {NILM }}$ and $\varepsilon_{F_{p}}{ }^{\text {NILM }}$ are defined:

$$
\begin{aligned}
& \varepsilon_{63}{ }^{\mathrm{NILM}}=\frac{\left|d_{63}{ }^{\mathrm{NLM}}\right|-\left|d_{63}{ }^{\mathrm{RHA}}\right|}{\left|d_{63}{ }^{\mathrm{RHA}}\right|} \\
& \varepsilon_{F_{p}}{ }^{\mathrm{NLM}}=\frac{\left|F_{p}^{\mathrm{NLM}}\right|-\left|F_{p}^{\mathrm{RHA}}\right|}{\left|F_{p}^{\mathrm{RHA}}\right|}
\end{aligned}
$$

where $\varepsilon_{63}{ }^{\text {NILM }}$ is the error of the middle-span nodal displacement given by the NILM to the RHA results, while $\varepsilon_{F_{p}}^{\text {NILM }}$ is that of the cable tension, similarly. In the right side of equation (41), $d_{63}{ }^{\text {NILM }}$ and $d_{63}{ }^{\text {RHA }}$ are the displacement of node 63 calculated by NILM and RHA, respectively. While in equation (42), $F_{p}{ }^{\text {NILM }}$ and $F_{p}{ }^{\text {RHA }}$ denote the cable tension of element 120 calculated by NILM and RHA, respectively. Here the cable tension refers to the member force caused by only seismic excitations, thus the pretension of the cable is excluded. The location of element 120 is marked in Fig.5.

Then, for the purpose of comparing the accuracy of the LMSM to that of the NILM, the error indexes $\varepsilon_{63}{ }^{\text {LMSM }}$ and $\varepsilon_{F_{p}}{ }^{\text {LMSM }}$ are defined by equation (43) and (44).

$$
\begin{aligned}
& \varepsilon_{63}{ }^{\text {LMSM }}=\frac{\left|d_{63}{ }^{\text {LMSM }}\right|-\left|d_{63}{ }^{\text {RHA }}\right|}{\left|d_{63}{ }^{\text {RHA }}\right|} \\
& \varepsilon_{F_{p}}^{\text {LMSM }}=\frac{\left|F_{p}^{{ }^{\text {LMSM }}}\right|-\left|F_{p}{ }^{\text {RHA }}\right|}{\left|F_{p}{ }^{\text {RHA }}\right|}
\end{aligned}
$$


The denotations of the parameters given in equation (43) and (44) are basically the same with that given in equation (41) and (42) besides of the differences between the superscripts “NILM", and “LMSM", indicating that the corresponding variables in the latter two equations are calculated by the LMSM rather than by the NILM. The values of $\varepsilon_{63}{ }^{\text {NILM }}$ and $\varepsilon_{63}{ }^{\text {LMSM }}$, as well as of $\varepsilon_{F_{p}}{ }^{\text {NILM }}$ and $\varepsilon_{F_{p}}{ }^{\text {LMSM }}$ for the sixteen earthquake inputs are plotted in Fig.14.

Fig.14. Values of $\varepsilon_{63}{ }^{\text {NILM }}, \varepsilon_{63}^{\text {LMSM }}$ and $\varepsilon_{F_{p}}{ }^{\text {NILM }}, \varepsilon_{F_{p}}{ }^{\text {LMSM }}$ for the 16 seismic waves As can be seen from the charts in Fig.14, the accuracy of the proposed NILM, shown either by $\varepsilon_{63}$ or by $\varepsilon_{F_{p}}$, is generally better than that of the LMSM as compared to the accurate RHA approach. For some of the seismic input situations, such as the $L w d \_90 \_n o r$, the Sfy_360_nor and the Snm_270_w excitations, the deviation of nodal displacement results given by the two approximate approaches are basically the same. Yet for their corresponding cable forces, the NILM gives better estimations than the LMSM does when the PGA of the seismic input reaches $1.0 \mathrm{~g}$ and $1.1 \mathrm{~g}$.

When the intensity degrees of the seismic excitations are relatively low, the discrepancies between $\varepsilon_{63}{ }^{\text {NILM }}$ and $\varepsilon_{63}{ }^{\text {LMSM }}$, as well as that between $\varepsilon_{F_{p}}{ }^{\text {NILM }}$ and $\varepsilon_{F_{p}}{ }^{\text {LMSM }}$ are pretty tiny. This is because that the structural deformation is not large enough for the cable net system to exhibit obvious nonlinearities. The differences between the values of $\varepsilon_{63}{ }^{\text {NILM }}$ and $\varepsilon_{63}{ }^{\text {LMSM }}$, as well as that between $\varepsilon_{F_{p}}{ }^{\text {NILM }}$ and $\varepsilon_{F_{p}}{ }^{\text {LMSM }}$ increases as the earthquake excitation becomes more severe. Under these situations, the cable net system experiences large deformations during the response histories, therefore, its geometrical stiffening property becomes unnegligible. Generally, the NILM gives better predictions of not only the nodal displacements but also the cable forces than the LMSM does, especially for severe seismic excitations when the PGAs are larger than $0.7 \mathrm{~g}$. This superiority of the NILM is mainly caused by its reasonable consideration of the geometrical nonlinearity of the cable 
net system.

Seeing from the charts in Fig.14, it could be found that the error indexes of cable forces, including $\varepsilon_{F_{p}}{ }^{\text {NILM }}$ and $\varepsilon_{F_{p}}{ }^{\text {LMSM }}$, show larger values than their counterparts $\varepsilon_{63}{ }^{\text {NILM }}$ and $\varepsilon_{63}{ }^{\text {LMSM }}$. As we know, in a finite element analysis process, the element strain which relates directly to the member force is computed as one order derivative of the nodal displacements, consequently, the error of displacement results tend to be amplified when the corresponding strain is educed. For the Cpc_16_nor, the Emc_90_w, the Sfpd_196, the Sfy_360_nor and the Sgs_ns_nor seismic excitations, the values of both $\varepsilon_{F_{p}}{ }^{\text {NILM }}$ and $\varepsilon_{F_{p}}{ }^{\text {LMSM }}$ are relatively large (up to more than $80 \%$ in some cases). As we know, the cable tensions are directly related to the strain of the cables in the cable axial direction, thus, unlike the error parameter $\varepsilon_{63}{ }^{\text {NILM }}$ and $\varepsilon_{63}{ }^{\text {LMSM }}$ which are determined by only the nodal displacements in the out-of-plane direction, the cable tensions are mainly effected by the nodal displacements in the in-plane-direction. Obviously, the in-plane nodal displacements are of very small values, as a result, even a small deviation between results given by different computing methods could lead to a large value of error.

Negative values of $\varepsilon_{F_{p}}$ given by the NILM appear in some cases such as those in which the $G p k \_360$, the Nrg_00_nor, the $S f \_69$ and the $S g s \_n s \_n o r$ earthquake excitations are employed. These results reveal one shortcoming of the NILM procedure, that is, underestimations of structural seismic responses would occasionally occur as NILM is adopted. Nevertheless, this shortcoming only appears when the PGAs of the excitations are excessively large. While for these situations, the seismic damage of the primary structural systems is of more concern than that of the cable net curtain walls.

One exception of the former discussion is that the under prediction of structural seismic responses appears through the whole PGA range of the Sun_10_nor seismic input when NILM is adopted. It is obviously not preferred for NILM to provide unconservative results which indicate unsafe 
calculations. But, the potential of the proposed NILM for evaluation of the nonlinear seismic responses of cable net structures should be sensed, seeing those good results provided by NILM as plotted in Fig.14. Encouraged by this potential, large scale of thorough numerical calculations, carried out on a set of normally designed cable net systems, are being executed by the authors. More insightful information that would be useful for further application of the NILM will be reported later.

\section{Conclusion}

The NILM developed in this paper aims at providing a simplified alternative approach for the nonlinear seismic response analysis of flexible cable net structures with irregular geometries. The NILM uses the stiffness parameter in generating the nonlinear ESDF systems for the dominant modes of the cable nets. And the nonlinear stiffness of an ESDF system is simulated by a quadratic function about the equivalent displacement. Based on a stochastic linearization process, the relationship between the equivalent linear stiffness and the displacement response of a modal ESDF system is established. Finally, the structural responses are computed according to the design response spectra through a non-iterative procedure.

The numerical example carried out on a trapezoid-shaped cable net supported curtain wall structure demonstrates that the proposed NILM, which takes the stiffness hardening effect into consideration, could not only yield better results than the LMSM does, but also exhibit satisfying timesaving property over the nonlinear RHA.

Because of the complex interaction between the seismic excitations and the nonlinear responses of the cable nets, underestimations of structural seismic responses would occasionally occur as NILM is adopted in a few cases. But the potential of NILM, as demonstrated by the numerical example given in this paper, should be emphasized. Accordingly, large scale parameter analyses are needed to illustrate the superiority and to provide the appropriate utilizing scope of the NILM, this undergoing study will be reported later.

\section{Acknowledgement}


Funding for this paper provided by the National Science Foundation of China under grant 51378379, is gratefully acknowledged. The authors' acknowledgment also goes to Miss Yiliang Zhou for the pictures she provided for Fig. 1 in this paper.

\section{Reference}

1. Memari AM, Behr RA, Kremer PA. Seismic behavior of curtain walls containing insulating glass units. J Archit Eng 2003; 9(2): 70-85.

2. Kwan ASK. A simple technique for calculating natural frequencies of geometrically nonlinear prestressed cable structures. Comput Struct 2000; 74(1): 41-50.

3. Vassilopoulou I, Gantes CJ. Vibration modes and natural frequencies of saddle form cable nets. Comput Struct 2010; 88(1): 105-19.

4. Vassilopoulou I, Gantes CJ. Nonlinear dynamic behavior of saddle-form cable nets under uniform harmonic load. Eng Struct 2011; 33(10): 2762-71.

5. Teich M, Warnstedt P, Gebbeken N. Influence of Negative Phase Loading on Cable Net Facade Response. J Archit Eng 2012; 18(4): 276-84.

6. Feng RQ, Wu Y, Shen SZ. Working mechanism of single-layer cable net supported glass curtain walls. Adv Struct Eng 2007; 10(2): 183-95.

7. Feng RQ, Wu Y, Shen SZ. A simplified calculating method of nonlinear frequency of cable net under mean wind load. Acta Mech Solida Sin 2006; 19(3): 248-54.

8. Feng RQ, Zhang LL, Wu Y, Shen SZ. Dynamic performance of cable net facades. J Constr Steel Res 2009; 65(12): 2217-27.

9. Feng RQ, Ye JH, Wu Y, Shen SZ. Nonlinear response spectra of cable net facades. Soil Dyn Earthq Eng 2012; 32(1): 71-86.

10. Wang Y, Wu L, Shi Y, Sun F, Luo K, Xu Y. FEM analysis and experimental study on monolayer cable net for glass facades: static performance. Adv Struct Eng 2007; 10(4): 371-82.

11. Shi Y, Wu L, Wang Y, Luo K, Xu Y. FEM analysis and experimental study on monolayer cable net for glass facades: dynamic properties. Adv Struct Eng 2007; 10(4): 383-95.

12. Wang L, Zhao Y. Non-linear planar dynamics of suspended cables investigated by the continuation technique. Eng Struct 2007; 29(6): 1135-44.

13. Yang L, Shi G, Yin H, Shi X, Guo Z. Full-scale experimental study on the influence of damages on the static behavior of the single-layer cable net structure. Struct 2015; http://dx.doi.org/10.1016/j.istruc.2015.04.002.

14. Feng RQ, Ye JH, Wu Y, Shen SZ. Nonlinear wind-resistant design method for cable net facades. Int J Struct Stab Dy 2011; 11(6): 1163-77.

15. Chopra AK. Dynamics of structures: theory and applications to earthquake engineering (Third Edition). Upper Saddle River, New Jersey, U.S.A.: Pearson Prentice Hall; 2007.

16. Naess A. Prediction of extreme response of nonlinear structures by extended stochastic linearization. Probabilist Eng Mech 1995; 10(3): 153-60.

17. Lin YY, Miranda E. Noniterative equivalent linear method for evaluation of existing structures. J Struct Eng-ASCE 2008; 134(11): 1685-95.

18. ASCE. Minimum design loads for buildings and other structures. ASCE/SEI 7-05-2006. 2006

19. EN 1998-1. Eurocode 8: Design of structures for earthquake resistance-Part 1: General rules, seismic actions and rules for buildings. 2005.

20. MOHURD \& AQSIQ of China. Code for seismic design of buildings. GB50011-2010, Beijing, China; 2010.

\section{Appendix A}

\section{A.1. ASCE/SEI 7-2005}

The design response spectrum given by the ASCE/SEI 7-05 document, named Minimum Design Loads for Buildings and Other Structures [18], is as follow: 
for $T<T_{0}$,

$$
S_{a}=S_{D S}\left(0.4+0.6 \frac{T}{T_{0}}\right)
$$

for $T_{0}<T \leq T_{S}$,

$$
S_{a}=S_{D S}
$$

for $T_{S}<T \leq T_{L}$

$$
S_{a}=\frac{S_{D 1}}{T}
$$

for $T_{L}<T$,

$$
S_{a}=\frac{S_{D 1} T_{L}}{T^{2}}
$$

where $S_{D S}$ is the design spectral response acceleration parameter at short periods, $S_{D 1}$ is the acceleration parameter at a period of $1 \mathrm{~s}, T$ is the fundamental period of the structure, $T_{0}=0.2 S_{D 1} / S_{D S}$, $T_{S}=S_{D 1} / S_{D S}$, and $T_{L}$ is the long-period transition period.

According to equation (x.x), the $T-S_{a}$ spectrum can be transformed into the following $d-k$ format, where $d$ indicates the displacement response and $k$ the structural stiffness:

for $k<\frac{4 \pi^{2} m}{T_{L}^{2}}$,

$$
d=\frac{S_{D 1} T_{L}}{4 \pi^{2}}
$$

for $\frac{4 \pi^{2} m}{T_{L}^{2}} \leq k<\frac{4 \pi^{2} m}{T_{S}^{2}}$,

$$
d=\frac{S_{D 1}}{2 \pi} \cdot \sqrt{\frac{m}{k}}
$$

for $\frac{4 \pi^{2} m}{T_{S}^{2}} \leq k<\frac{4 \pi^{2} m}{T_{0}^{2}}$,

$$
d=\frac{m S_{D S}}{k}
$$

for $\frac{4 \pi^{2} m}{T_{0}^{2}} \leq k$

$$
d=\left[0.4+\frac{1.2 \pi}{T_{0}} \cdot \sqrt{\frac{m}{k}}\right] \cdot \frac{m S_{D S}}{k}
$$

\section{A.2. Eurocode 8 EN 1998-1:2004}

The horizontal elastic response spectrum given by the Eurocode 8: Design of structures for earthquake resistance [19], is as follow:

for $0<T \leq T_{B}$, 


$$
S_{e}(T)=a_{g} S\left[1+\frac{T}{T_{B}}(2.5 \eta-1)\right]
$$

for $T_{\mathrm{B}}<T \leq T_{C}$,

$$
S_{e}(T)=2.5 \eta a_{g} S
$$

for $T_{\mathrm{C}}<T \leq T_{D}$,

$$
S_{e}(T)=2.5 \eta a_{g} S\left(\frac{T_{C}}{T}\right)
$$

for $T_{\mathrm{D}}<T \leq 4 \mathrm{~s}$,

$$
S_{e}(T)=2.5 \eta a_{g} S\left(\frac{T_{C} T_{D}}{T^{2}}\right)
$$

where $S_{e}(T)$ is the elastic response spectrum, $T$ is the vibration period of a linear SDF system, $a_{g}$ is the design ground acceleration on type A ground, $T_{B}$ and $T_{C}$ are the lower and upper limit of the period of the constant spectral acceleration branch, respectively, $T_{D}$ is the value defining the beginning of the constant displacement response range of the spectrum, $S$ is the soil factor, and $\eta$ is the damping correction factor.

The corresponding $d-k$ format spectrum is:

for $\frac{4 \pi^{2} m}{T_{B}^{2}} \leq k$,

$$
d=\frac{m a_{g} S}{k}\left[1+\frac{2 \pi}{T_{B}} \sqrt{\frac{m}{k}}(2.5 \eta-1)\right]
$$

for $\frac{4 \pi^{2} m}{T_{C}^{2}} \leq k<\frac{4 \pi^{2} m}{T_{B}^{2}}$,

$$
d=2.5 \eta \frac{m a_{g} S}{k}
$$

for $\frac{4 \pi^{2} m}{T_{D}^{2}} \leq k<\frac{4 \pi^{2} m}{T_{C}^{2}}$,

$$
d=1.25 \eta T_{C} \frac{a_{g} S}{\pi} \sqrt{\frac{m}{k}}
$$

for $\frac{\pi^{2} m}{4} \leq k<\frac{4 \pi^{2} m}{T_{D}^{2}}$,

$$
d=0.625 \eta T_{C} T_{D} \frac{a_{g} S}{\pi^{2}}
$$

\section{A.3. China GB50011-2010}

The elastic acceleration response spectrum proposed by the Chinese code for seismic design of buildings [20], is as follow:

for $0<T \leq 0.1 \mathrm{~s}$, 


$$
a=\left[\left(10 \eta_{2}-4.5\right) T+0.45\right] \alpha_{\max } g
$$

for $0.1 \mathrm{~s}<T \leq T_{g}$,

$$
a=\eta_{2} \alpha_{\max } g
$$

for $T_{\mathrm{g}}<T \leq 5 T_{g}$,

$$
a=\left(\frac{T_{g}}{T}\right)^{\gamma} \eta_{2} \alpha_{\max } g
$$

for $5 T_{\mathrm{g}}<T \leq 6 \mathrm{~s}$,

$$
a=\left[0.2^{\gamma} \eta_{2}-\eta_{1}\left(T-5 T_{g}\right)\right] \alpha_{\max } g
$$

where $a$ is the acceleration response, $g$ is gravity, $\alpha_{\max }$ is the peak seismic effect coefficient, $\eta_{1}$ and $\eta_{2}$ are adjusting coefficients relevant to the structural damping ratio. The index $\gamma$, also noted as the decaying index of the spectrum value, is determined by the damping ratio, and $T$ is the period of the structure while $T_{g}$ means the characteristic period of the building site.

The corresponding $d-k$ format spectrum is:

for $\frac{4 \pi^{2} m}{0.01} \leq k$,

$$
d=\left[2 \pi\left(10 \eta_{2}-4.5\right) \sqrt{\frac{m}{k}}+0.45\right] \frac{m g \alpha_{\max }}{k}
$$

for $\frac{4 \pi^{2} m}{T_{g}^{2}} \leq k<\frac{4 \pi^{2} m}{0.01}$,

$$
d=\frac{\eta_{2} m g \alpha_{\max }}{k}
$$

for $\frac{4 \pi^{2} m}{25 T_{g}^{2}} \leq k<\frac{4 \pi^{2} m}{T_{g}^{2}}$,

$$
d=\left(\frac{T_{g}}{2 \pi} \sqrt{\frac{k}{m}}\right)^{\gamma} \frac{\eta_{2} m g \alpha_{\max }}{k}
$$

for $\frac{4 \pi^{2} m}{36} \leq k<\frac{4 \pi^{2} m}{25 T_{g}^{2}}$,

$$
d=\left(0.2^{\gamma} \eta_{2}-2 \pi \eta_{1} \sqrt{\frac{m}{k}}+5 T_{g} \eta_{1}\right) \frac{m g \alpha_{\max }}{k}
$$


Table 1 Information of the dominant vibration modes and the corresponding ESDF systems

\begin{tabular}{cccccccc}
\hline \multirow{2}{*}{ Mode } & $T(\mathrm{~s})$ & \multirow{2}{*}{$E M F$} & MPF & $k_{n}{ }^{*}(\mathrm{~N} / \mathrm{mm})$ & $m_{n}{ }^{*}(\mathrm{t})$ & \multicolumn{2}{c}{ Coefficients in the $k_{n}{ }^{*}$ expression } \\
\cline { 7 - 8 } & & & & & & $a_{0}$ & $b_{0}$ \\
\hline 1 & 0.36251 & 0.72619 & 2.37800 & 18.77877 & 0.06251 & $4.64909 \times 10^{-4}$ & 18.77877 \\
3 & 0.21281 & 0.06085 & 0.68840 & 54.54106 & 0.06257 & $2.39132 \times 10^{-4}$ & 54.54106 \\
8 & 0.14981 & 0.05868 & 0.67597 & 110.04750 & 0.06256 & $2.06093 \times 10^{-3}$ & 110.04750 \\
\hline
\end{tabular}

Table 2 List of the earthquake excitations

\begin{tabular}{cccccccc}
\hline NO. & $\begin{array}{c}\text { Earthquake } \\
\text { Excitation }\end{array}$ & $\begin{array}{c}\text { PGA } \\
(\mathrm{g})\end{array}$ & $\begin{array}{c}\text { Predominant } \\
\text { Period }(\mathrm{s})\end{array}$ & $\begin{array}{c}\text { Duration } \\
(\mathrm{s})\end{array}$ & $\begin{array}{c}\text { Location of } \\
\text { Recording Station }\end{array}$ & Earthquake & Year \\
\hline 1 & Bc_310 & 0.595 & 0.44 & 37.800 & Bonds Corner, El Centro & Imperial Valley & 1979 \\
2 & Cau_ns_a & 0.055 & 0.46 & 21.405 & Cauquenes & Central Chile & 1985 \\
3 & Cpc_16_nor & 0.389 & 0.60 & 55.580 & Topanga Canyon BLVD. & Northridge & 1994 \\
4 & Emc_90_w & 0.237 & 0.32 & 28.280 & Fairview Ave. & Whittier Narrows & 1987 \\
5 & Fcy_90_1 & 0.283 & 0.66 & 60.000 & Foster City & Loma Prieta & 1989 \\
6 & Gpk_360w & 0.124 & 0.52 & 30.470 & Griffith Park & Whittier Narrows & 1987 \\
7 & Lwd_90_nor & 0.122 & 0.24 & 35.400 & Del Amo BLVD. & Northridge & 1994 \\
8 & Nrg_00_nor & 0.453 & 0.40 & 60.240 & Saticoy ST. & Northridge & 1994 \\
9 & Sf_69 & 0.315 & 0.32 & 61.820 & San Fernando & San Fernando & 1971 \\
10 & Sfpd_196 & 1.075 & 0.42 & 41.580 & Pocoima Dam & San Fernando & 1971 \\
11 & Sfy_360_nor & 0.308 & 0.32 & 60.000 & Nordhoff Ave Fire Station & Northridge & 1994 \\
12 & Sgs_ns_nor & 0.138 & 0.14 & 45.976 & San Gabriel & Northridge & 1994 \\
13 & Snm_270_w & 0.139 & 0.12 & 40.000 & San Marino & Whittier Narrows & 1987 \\
14 & Sun_10_nor & 0.130 & 0.56 & 49.900 & Gleason Ave., Sunland & Northridge & 1994 \\
15 & Tar_90_nor & 1.780 & 0.34 & 60.020 & Cedar Hill & Northridge & 1994 \\
16 & Tri_90_1 & 0.159 & 0.62 & 40.020 & Treasure Island & Loma Prieta & 1989 \\
\hline
\end{tabular}


Table $3 S L F$ s of the $1^{\text {st }}$ mode corresponding to the displacements of the ESDF systems

\begin{tabular}{|c|c|c|c|c|c|c|c|c|c|c|}
\hline \multirow{2}{*}{$\begin{array}{l}\text { Earthquake } \\
\text { Excitation }\end{array}$} & \multirow{2}{*}{$\begin{array}{l}\text { Analysis } \\
\text { Approach }\end{array}$} & \multicolumn{9}{|c|}{ PGA of the seismic input (g) } \\
\hline & & 0.3 & 0.4 & 0.5 & 0.6 & 0.7 & 0.8 & 0.9 & 1.0 & 1.1 \\
\hline \multirow{2}{*}{ Bc_310 } & LMSM & 1233.4 & 1651.5 & 2081.5 & 2515.7 & 2960.5 & 3422.6 & 3893.4 & 4379.4 & 4889.9 \\
\hline & NILM & 1233.4 & 1651.5 & 2062.5 & 2413.9 & 2699.9 & 3104.4 & 3498.9 & 3736.6 & 3966.6 \\
\hline \multirow{2}{*}{ Cau_ns_a } & LMSM & 1977.5 & 2670.8 & 3397.1 & 4153.7 & 4952.5 & 5800.2 & 6703.0 & 7667.8 & 8701.4 \\
\hline & NILM & 1903.0 & 2564.2 & 3254.6 & 3956.1 & 4368.6 & 5049.2 & 5413.8 & 6110.9 & 6877.6 \\
\hline \multirow{2}{*}{ Cpc_16_nor } & LMSM & 1536.1 & 2062.5 & 2602.9 & 3159.0 & 3736.6 & 4341.8 & 4969.6 & 5625.4 & 6315.8 \\
\hline & NILM & 1536.1 & 2062.5 & 2602.9 & 3159.0 & 3736.6 & 4245.0 & 4550.1 & 4721.5 & 4884.2 \\
\hline \multirow{2}{*}{ Emc_90_w } & LMSM & 2109.9 & 2851.3 & 3632.1 & 4450.6 & 5320.1 & 6247.5 & 7246.9 & 8315.3 & 9465.2 \\
\hline & NILM & 2109.9 & 2851.3 & 3632.1 & 4450.6 & 5320.1 & 6098.5 & 6607.1 & 7022.2 & 7307.7 \\
\hline \multirow{2}{*}{ Fcy_90_1 } & LMSM & 1297.6 & 1740.0 & 2190.6 & 2646.6 & 3119.3 & 3605.9 & 4110.7 & 4633.1 & 5174.4 \\
\hline & NILM & 1297.6 & 1735.3 & 2181.1 & 2636.9 & 3039.9 & 3442.9 & 3825.5 & 4116.1 & 4384.7 \\
\hline \multirow{2}{*}{ Gpk_360w } & LMSM & 1119.8 & 1499.4 & 1884.4 & 2276.0 & 2675.6 & 3084.5 & 3504.0 & 3935.2 & 4379.4 \\
\hline & NILM & 1097.1 & 1448.9 & 1786.6 & 2105.2 & 2409.1 & 2685.3 & 2940.6 & 3163.9 & 3361.5 \\
\hline \multirow{2}{*}{ Lwd_90_nor } & LMSM & 1554.4 & 2091.0 & 2636.9 & 3203.7 & 3794.1 & 4406.4 & 5043.5 & 5715.8 & 6421.9 \\
\hline & NILM & 1554.4 & 2091.0 & 2636.9 & 3203.7 & 3794.1 & 4406.4 & 5043.5 & 5571.9 & 6185.4 \\
\hline \multirow{2}{*}{ Nrg_00_nor } & LMSM & 1730.7 & 2328.2 & 2945.6 & 3585.4 & 4255.8 & 4958.2 & 5703.8 & 6485.8 & 7314.5 \\
\hline & NILM & 1716.7 & 2314.0 & 2925.8 & 3565.1 & 4223.6 & 4782.3 & 5255.6 & 5583.6 & 5920.8 \\
\hline \multirow{2}{*}{ Sf_69 } & LMSM & 1721.3 & 2318.7 & 2930.7 & 3570.2 & 4239.7 & 4935.4 & 5673.6 & 6447.4 & 7273.9 \\
\hline & NILM & 1721.3 & 2318.7 & 2915.8 & 3432.7 & 3961.3 & 4522.5 & 4952.5 & 5320.1 & 5577.8 \\
\hline \multirow{2}{*}{ Sfpd_196 } & LMSM & 1156.0 & 1549.8 & 1949.6 & 2356.7 & 2772.6 & 3193.7 & 3632.1 & 4083.8 & 4550.1 \\
\hline & NILM & 1156.0 & 1549.8 & 1949.6 & 2356.7 & 2748.3 & 3015.1 & 3305.5 & 3529.4 & 3668.7 \\
\hline \multirow{2}{*}{ Sfy_360_nor } & LMSM & 1435.2 & 1926.3 & 2428.4 & 2945.6 & 3473.5 & 4024.7 & 4599.9 & 5197.1 & 5824.3 \\
\hline & NILM & 1435.2 & 1926.3 & 2428.4 & 2945.6 & 3473.5 & 4024.7 & 4599.9 & 5145.9 & 5495.8 \\
\hline \multirow{2}{*}{ Sgs_ns_nor } & LMSM & 1753.9 & 2356.7 & 2985.3 & 3637.3 & 4320.3 & 5037.8 & 5794.2 & 6588.0 & 7436.1 \\
\hline & NILM & 1753.9 & 2356.7 & 2985.3 & 3504.0 & 3778.4 & 3977.0 & 4121.5 & 4374.0 & 4878.5 \\
\hline \multirow{2}{*}{ Snm_270_w } & LMSM & 1142.4 & 1531.5 & 1921.6 & 2323.5 & 2733.8 & 3149.1 & 3580.3 & 4024.7 & 4483.8 \\
\hline & NILM & 1142.4 & 1531.5 & 1921.6 & 2323.5 & 2729.0 & 3139.1 & 3504.0 & 3825.5 & 4153.7 \\
\hline \multirow{2}{*}{ Sun_10_nor } & LMSM & 1698.1 & 2285.5 & 2891.0 & 3519.3 & 4175.2 & 4861.5 & 5583.6 & 6346.8 & 7153.6 \\
\hline & NILM & 1494.8 & 1912.3 & 2409.1 & 2920.8 & 3453.1 & 3997.9 & 4489.3 & 4760.2 & 4924.0 \\
\hline \multirow{2}{*}{ Tar_90_nor } & LMSM & 1499.4 & 2015.0 & 2539.9 & 3084.5 & 3642.5 & 4228.9 & 4838.7 & 5472.3 & 6142.0 \\
\hline & NILM & 1499.4 & 2015.0 & 2539.9 & 3084.5 & 3642.5 & 4180.6 & 4450.6 & 4688.3 & 5163.0 \\
\hline \multirow{2}{*}{ Tri_90_1 } & LMSM & 1549.8 & 2081.5 & 2627.2 & 3188.8 & 3773.2 & 4379.4 & 5020.8 & 5685.7 & 6384.0 \\
\hline & NILM & 1549.8 & 2081.5 & 2627.2 & 3188.8 & 3773.2 & 4379.4 & 4798.9 & 5249.8 & 5625.4 \\
\hline
\end{tabular}


Table 4 Linear $S L F$ s of the $3^{\text {rd }}$ and the $8^{\text {th }}$ mode corresponding to the displacements of the ESDF systems

\begin{tabular}{|c|c|c|c|c|c|c|c|c|c|c|}
\hline \multirow{2}{*}{$\begin{array}{l}\text { Earthquake } \\
\text { Excitation }\end{array}$} & \multirow{2}{*}{$\begin{array}{l}\text { Analysis } \\
\text { Approach }\end{array}$} & \multicolumn{9}{|c|}{ PGA of the seismic input ( $g$ ) } \\
\hline & & 0.3 & 0.4 & 0.5 & 0.6 & 0.7 & 0.8 & 0.9 & 1.0 & 1.1 \\
\hline \multirow{2}{*}{ Bc_310 } & Mode 3 & 311.8 & 417.0 & 518.5 & 623.8 & 729.1 & 834.5 & 936.2 & 1041.7 & 1147.2 \\
\hline & Mode 8 & 334.9 & 446.6 & 558.4 & 670.2 & 782.0 & 893.9 & 1006.0 & 1118.0 & 1230.2 \\
\hline \multirow{2}{*}{ Cau_ns_a } & Mode 3 & 270.5 & 360.6 & 450.8 & 541.1 & 631.3 & 717.8 & 808.1 & 898.5 & 989.0 \\
\hline & Mode 8 & 171.2 & 230.7 & 282.8 & 342.4 & 401.9 & 454.1 & 513.7 & 573.3 & 625.5 \\
\hline \multirow{2}{*}{ Cpc_16_nor } & Mode 3 & 356.9 & 477.2 & 597.5 & 717.8 & 838.2 & 955.0 & 1075.6 & 1196.2 & 1317.1 \\
\hline & Mode 8 & 290.3 & 379.6 & 476.4 & 573.3 & 670.2 & 767.1 & 864.1 & 953.7 & 1050.8 \\
\hline \multirow{2}{*}{ Emc_90_w } & Mode 3 & 349.4 & 465.9 & 582.4 & 699.0 & 815.6 & 932.4 & 1049.2 & 1166.1 & 1283.1 \\
\hline & Mode 8 & 297.7 & 401.9 & 498.8 & 595.7 & 700.0 & 796.9 & 901.4 & 998.5 & 1095.6 \\
\hline \multirow{2}{*}{ Fcy_90_1 } & Mode 3 & 285.5 & 379.4 & 473.4 & 567.4 & 665.2 & 759.2 & 853.3 & 947.5 & 1045.5 \\
\hline & Mode 8 & 230.7 & 305.2 & 387.0 & 461.5 & 536.0 & 618.0 & 692.5 & 767.1 & 849.1 \\
\hline \multirow{2}{*}{ Gpk_360w } & Mode 3 & 465.9 & 620.0 & 774.2 & 928.7 & 1086.9 & 1241.5 & 1396.5 & 1551.5 & 1710.7 \\
\hline & Mode 8 & 282.8 & 372.1 & 469.0 & 558.4 & 655.3 & 744.7 & 841.7 & 931.3 & 1028.4 \\
\hline \multirow{2}{*}{ Lwd_90_nor } & Mode 3 & 687.7 & 917.4 & 1147.2 & 1377.6 & 1608.2 & 1839.7 & 2071.6 & 2300.5 & 2533.9 \\
\hline & Mode 8 & 439.2 & 588.2 & 737.2 & 879.0 & 1028.4 & 1177.8 & 1327.6 & 1470.0 & 1620.0 \\
\hline \multirow{2}{*}{ Nrg_00_nor } & Mode 3 & 368.1 & 492.2 & 612.5 & 736.6 & 860.8 & 981.4 & 1105.8 & 1230.2 & 1351.2 \\
\hline & Mode 8 & 282.8 & 372.1 & 469.0 & 558.4 & 655.3 & 744.7 & 841.7 & 931.3 & 1028.4 \\
\hline \multirow{2}{*}{ Sf_69 } & Mode 3 & 390.7 & 522.3 & 650.1 & 781.7 & 909.8 & 1041.7 & 1173.6 & 1302.0 & 1434.3 \\
\hline & Mode 8 & 387.0 & 521.1 & 647.8 & 782.0 & 908.9 & 1043.3 & 1170.3 & 1305.1 & 1432.5 \\
\hline \multirow{2}{*}{ Sfpd_196 } & Mode 3 & 304.3 & 405.7 & 507.2 & 605.0 & 706.5 & 808.1 & 909.8 & 1011.6 & 1113.3 \\
\hline & Mode 8 & 320.0 & 424.3 & 528.6 & 640.4 & 744.7 & 849.1 & 961.2 & 1065.7 & 1170.3 \\
\hline \multirow{2}{*}{ Sfy_360_nor } & Mode 3 & 424.5 & 567.4 & 710.3 & 853.3 & 992.7 & 1135.9 & 1279.3 & 1423.0 & 1562.8 \\
\hline & Mode 8 & 267.9 & 357.3 & 446.6 & 536.0 & 625.5 & 714.9 & 804.3 & 893.9 & 976.1 \\
\hline \multirow{2}{*}{ Sgs_ns_nor } & Mode 3 & 714.1 & 951.3 & 1188.7 & 1426.8 & 1665.2 & 1908.0 & 2147.9 & 2388.2 & 2629.9 \\
\hline & Mode 8 & 767.1 & 1028.4 & 1282.6 & 1545.0 & 1800.7 & 2064.6 & 2322.1 & 2588.3 & 2847.9 \\
\hline \multirow{2}{*}{ Snm_270_w } & Mode 3 & 308.0 & 409.5 & 511.0 & 612.5 & 714.1 & 819.4 & 921.1 & 1022.9 & 1124.6 \\
\hline & Mode 8 & 379.6 & 498.8 & 625.5 & 752.1 & 879.0 & 1006.0 & 1133.0 & 1252.6 & 1380.1 \\
\hline \multirow{2}{*}{ Sun_10_nor } & Mode 3 & 405.7 & 541.1 & 680.2 & 815.6 & 951.3 & 1086.9 & 1222.6 & 1358.7 & 1494.8 \\
\hline & Mode 8 & 506.2 & 670.2 & 841.7 & 1006.0 & 1177.8 & 1342.6 & 1515.0 & 1687.8 & 1853.4 \\
\hline \multirow{2}{*}{ Tar_90_nor } & Mode 3 & 296.8 & 394.4 & 496.0 & 593.7 & 691.5 & 793.0 & 891.0 & 989.0 & 1090.7 \\
\hline & Mode 8 & 267.9 & 349.8 & 439.2 & 528.6 & 618.0 & 707.4 & 796.9 & 879.0 & 968.6 \\
\hline \multirow{2}{*}{ Tri_90_1 } & Mode 3 & 210.4 & 278.0 & 349.4 & 417.0 & 488.4 & 556.1 & 627.6 & 695.3 & 766.7 \\
\hline & Mode 8 & 238.2 & 320.0 & 394.5 & 476.4 & 558.4 & 632.9 & 714.9 & 796.9 & 871.5 \\
\hline
\end{tabular}




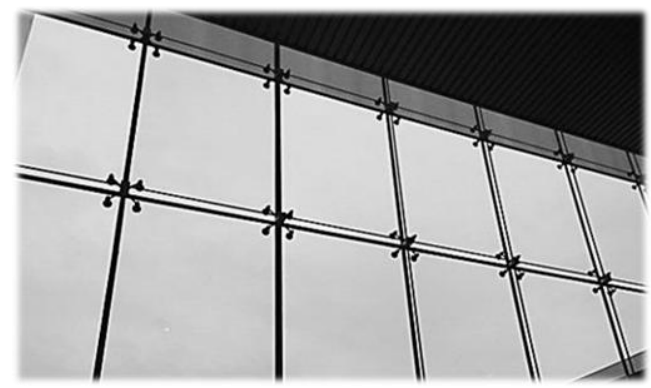

Inside view of the B Tower of Baoland Plaza, Shanghai, China

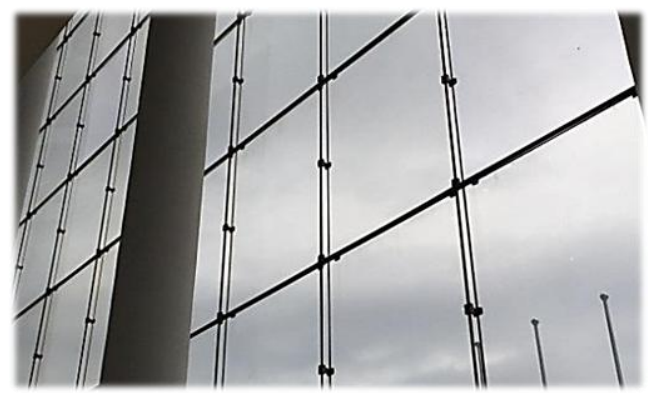

Inside view of the Siemens Center, Shanghai, China

Fig.1. Cable net systems

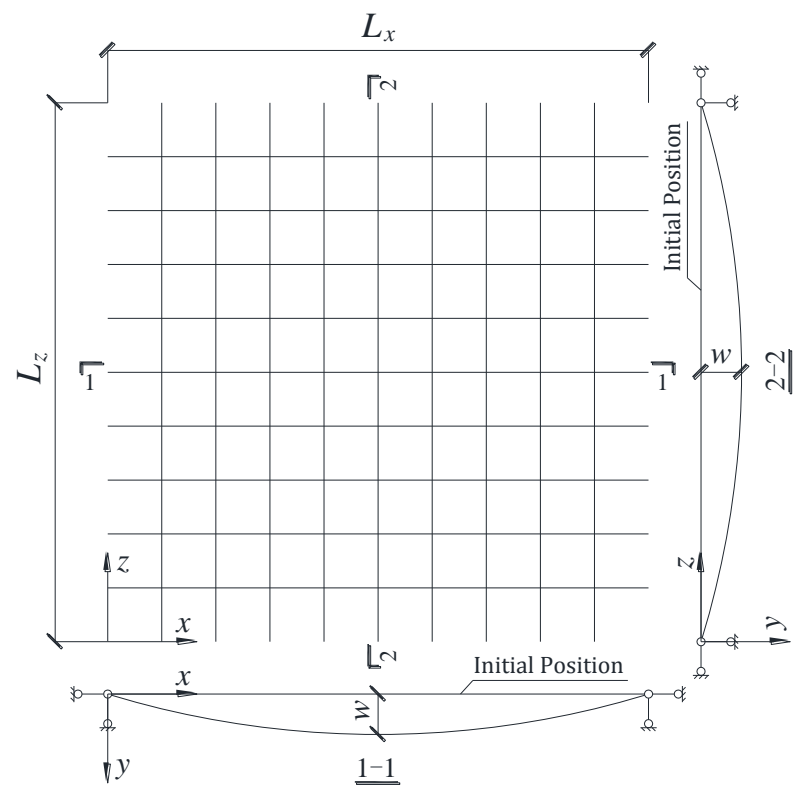

Fig.2. Deformation pattern of the rectangular cable net in Feng's approach [9] 


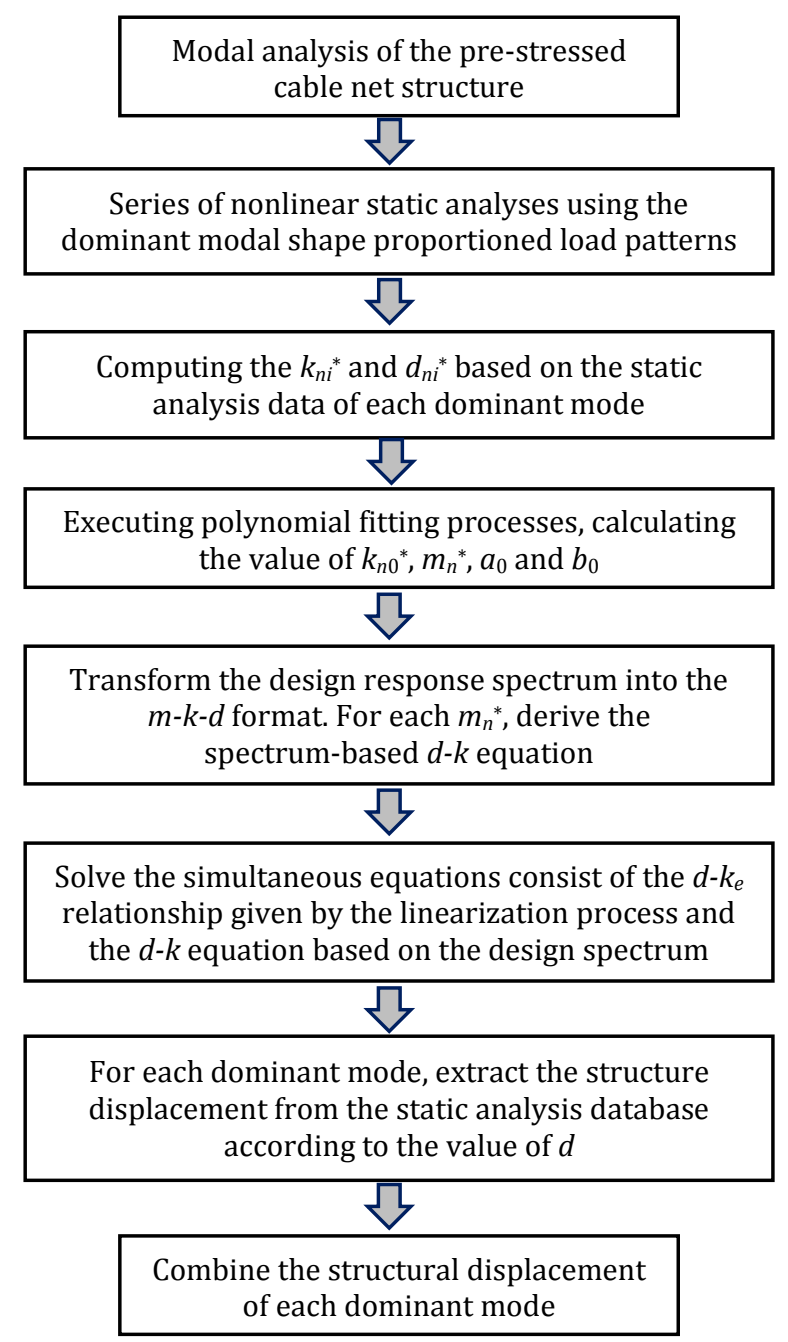

Fig.3. The non-iterative linearized analysis procedure

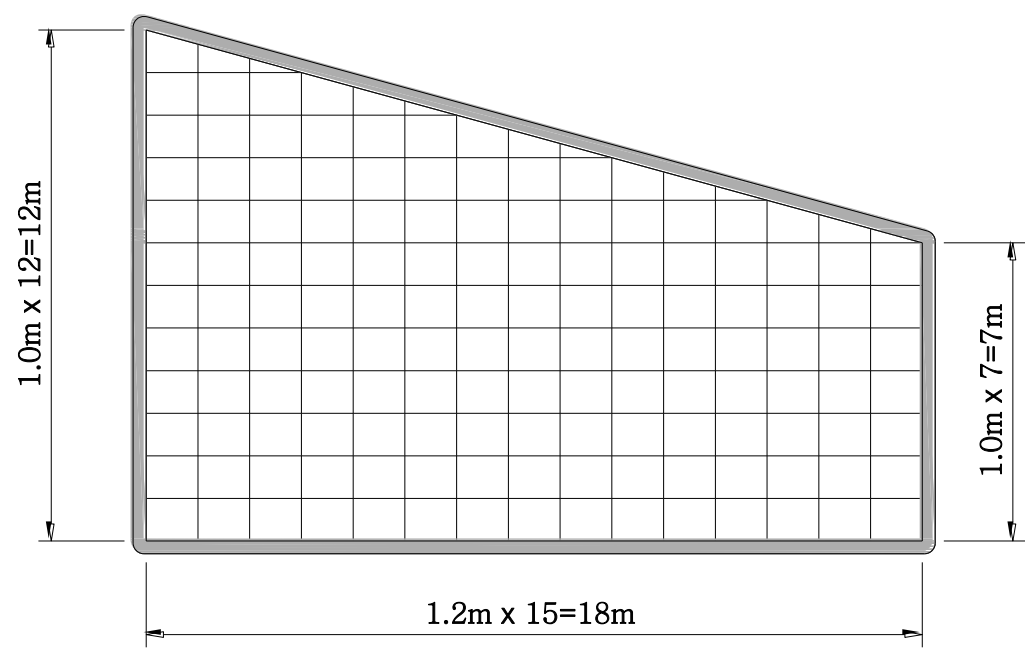

Fig.4. Elevation of the trapezoidal cable net supported curtain wall system 


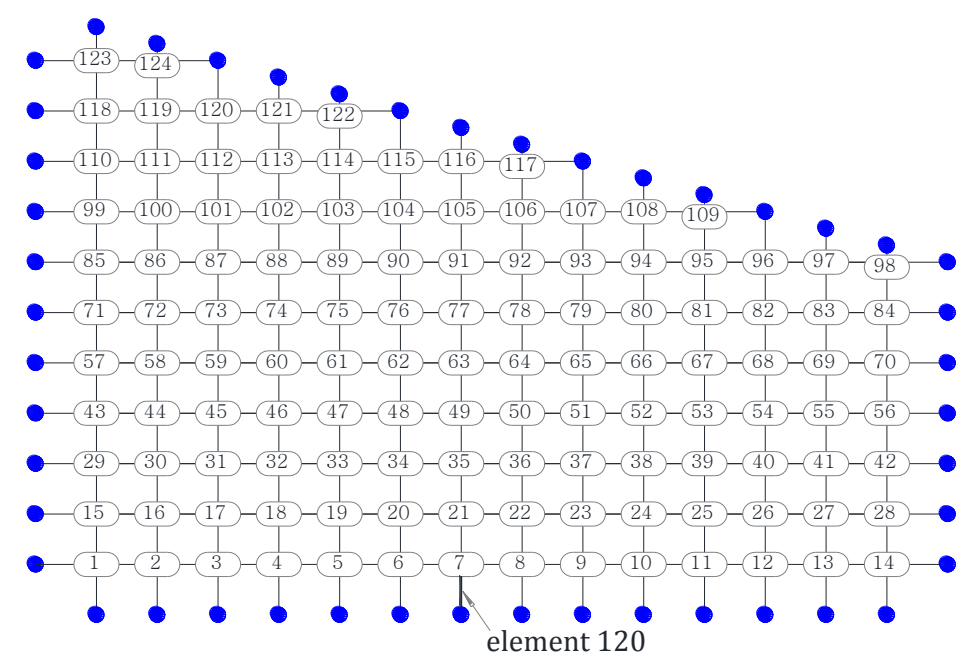

Fig.5. Node numbers of the FEM of the cable net

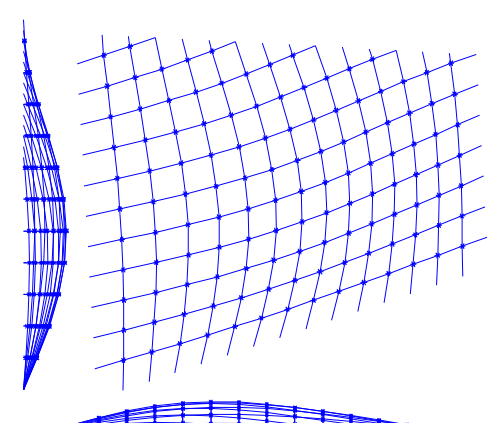

Mode 1

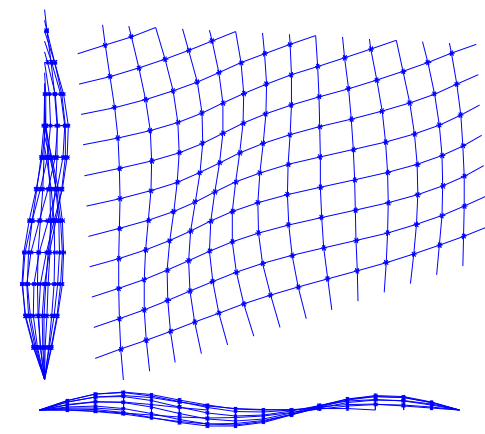

Mode 3
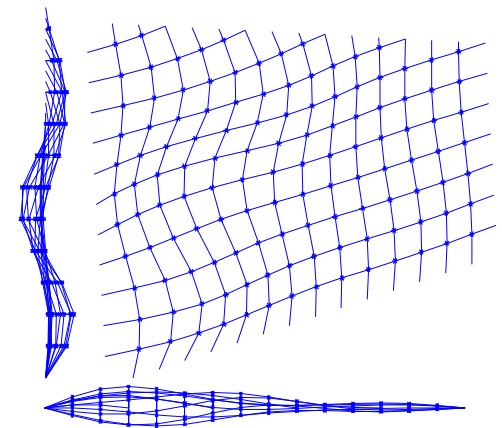

Mode 8

Fig.6. Dominant vibration modes of the cable net supported curtain wall model
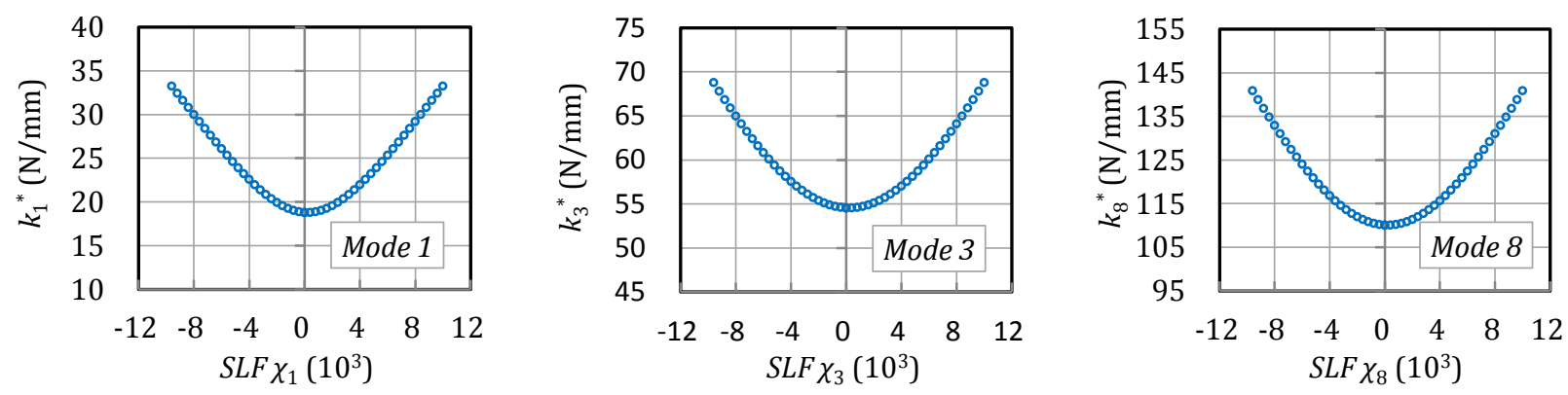

Fig.7. Relationship between $\chi_{n}$ and $k_{n}{ }^{*}$ 

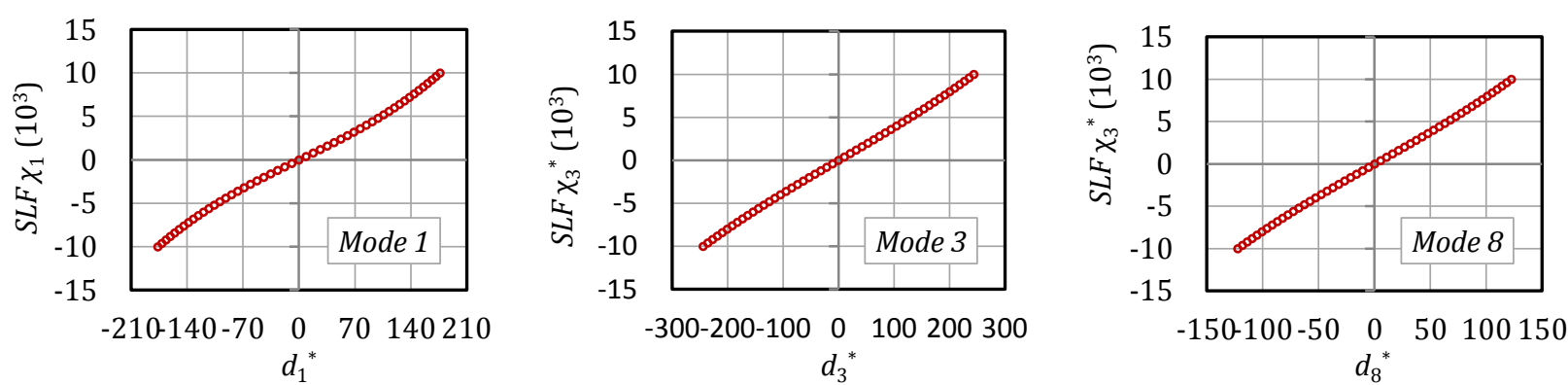

Fig.8. The equivalent load-displacement curves of the dominant vibration modes
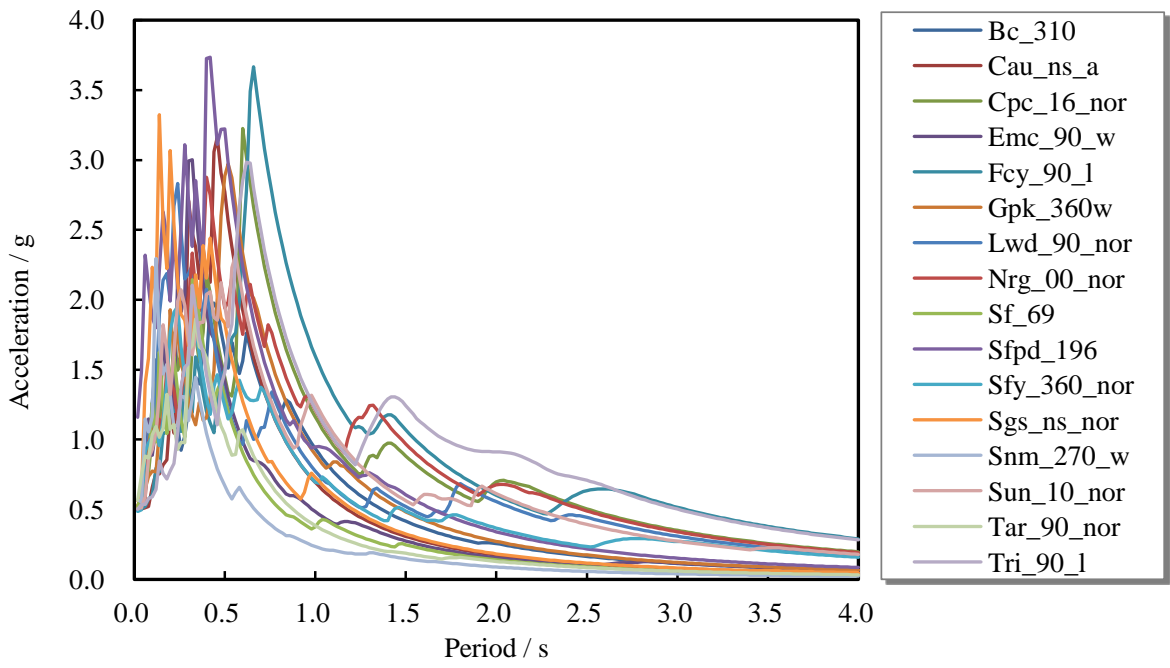

a. Acceleration response spectra

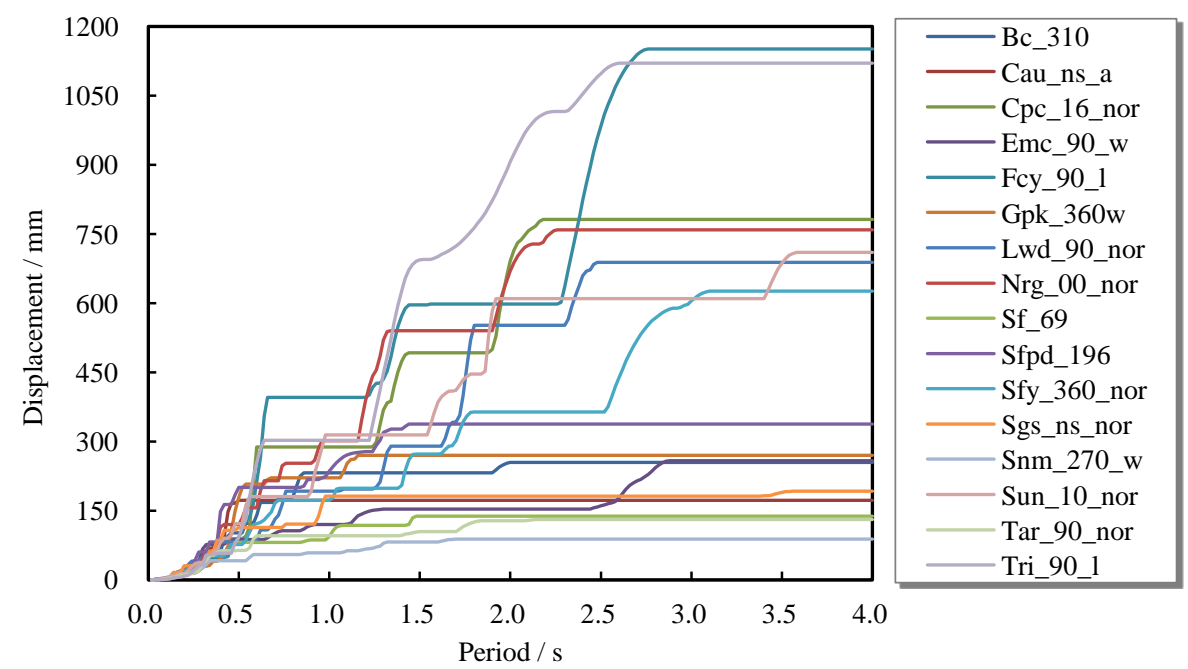

b. Displacement response spectra

Fig.9. Linear response spectra of the seismic waves 

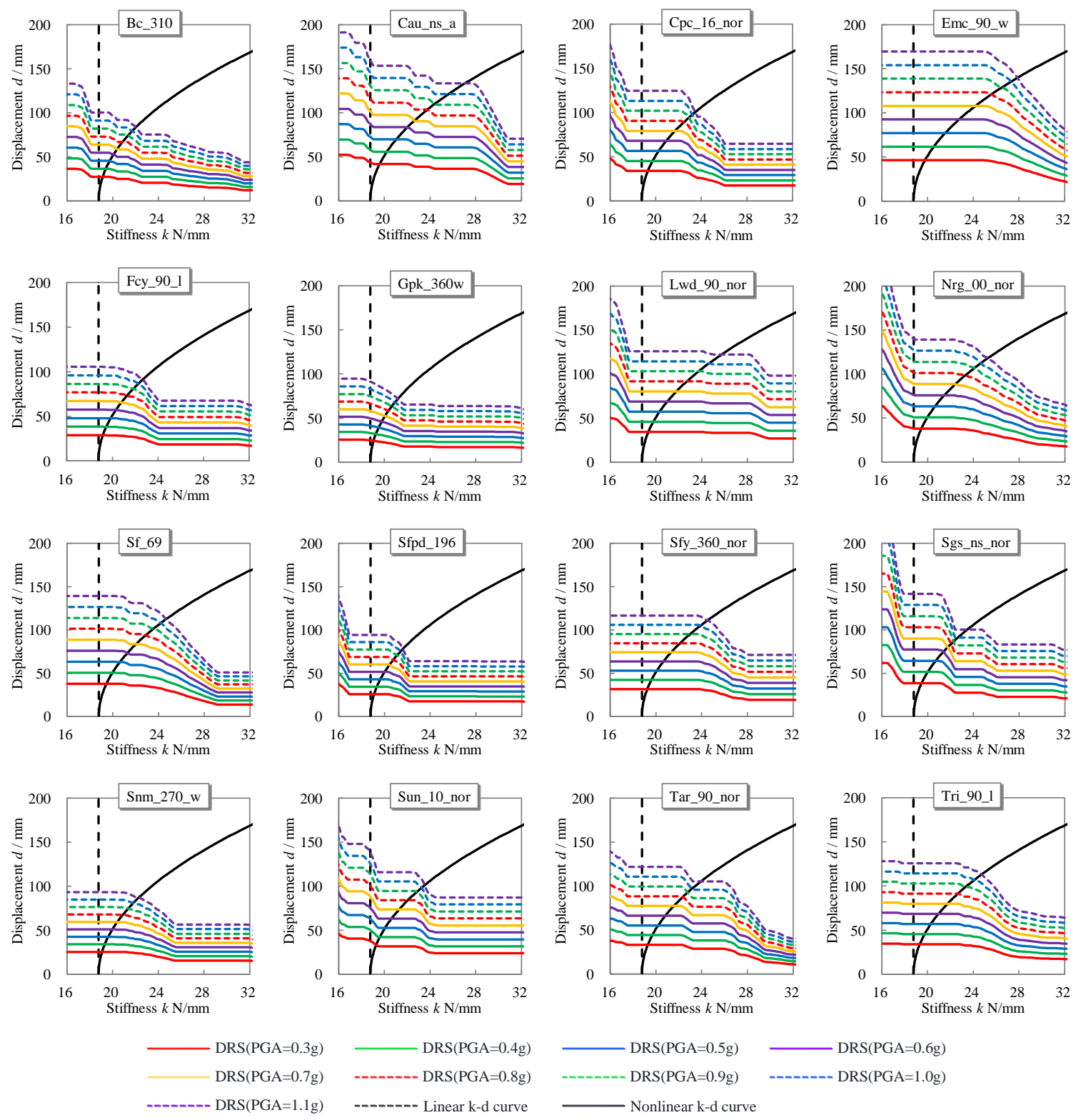

Fig.10. Graphic solutions for the ESDF responses of the $1^{\text {st }}$ mode 

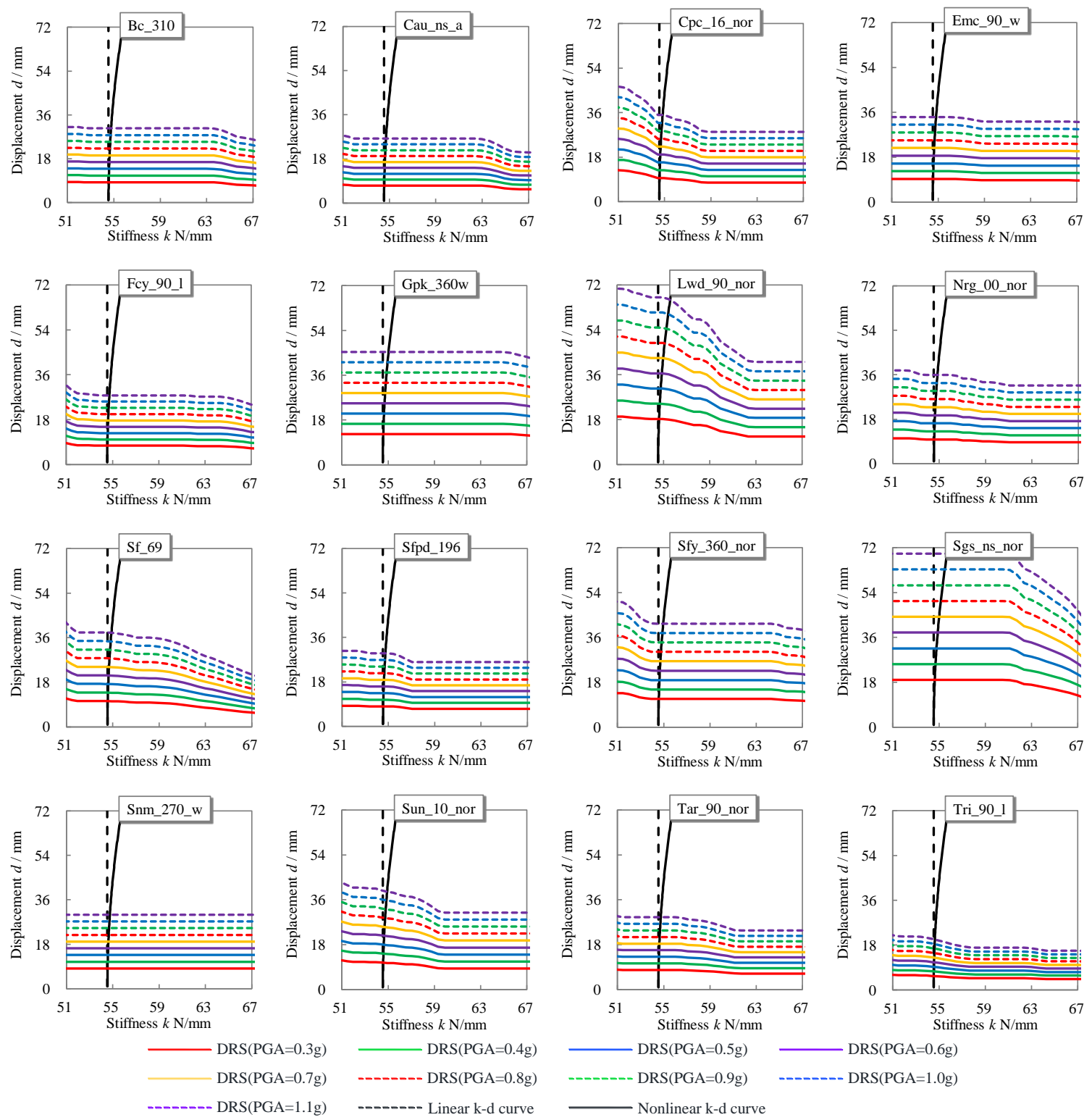

Fig.11. Graphic solutions for the ESDF responses of the $3^{\text {rd }}$ mode 

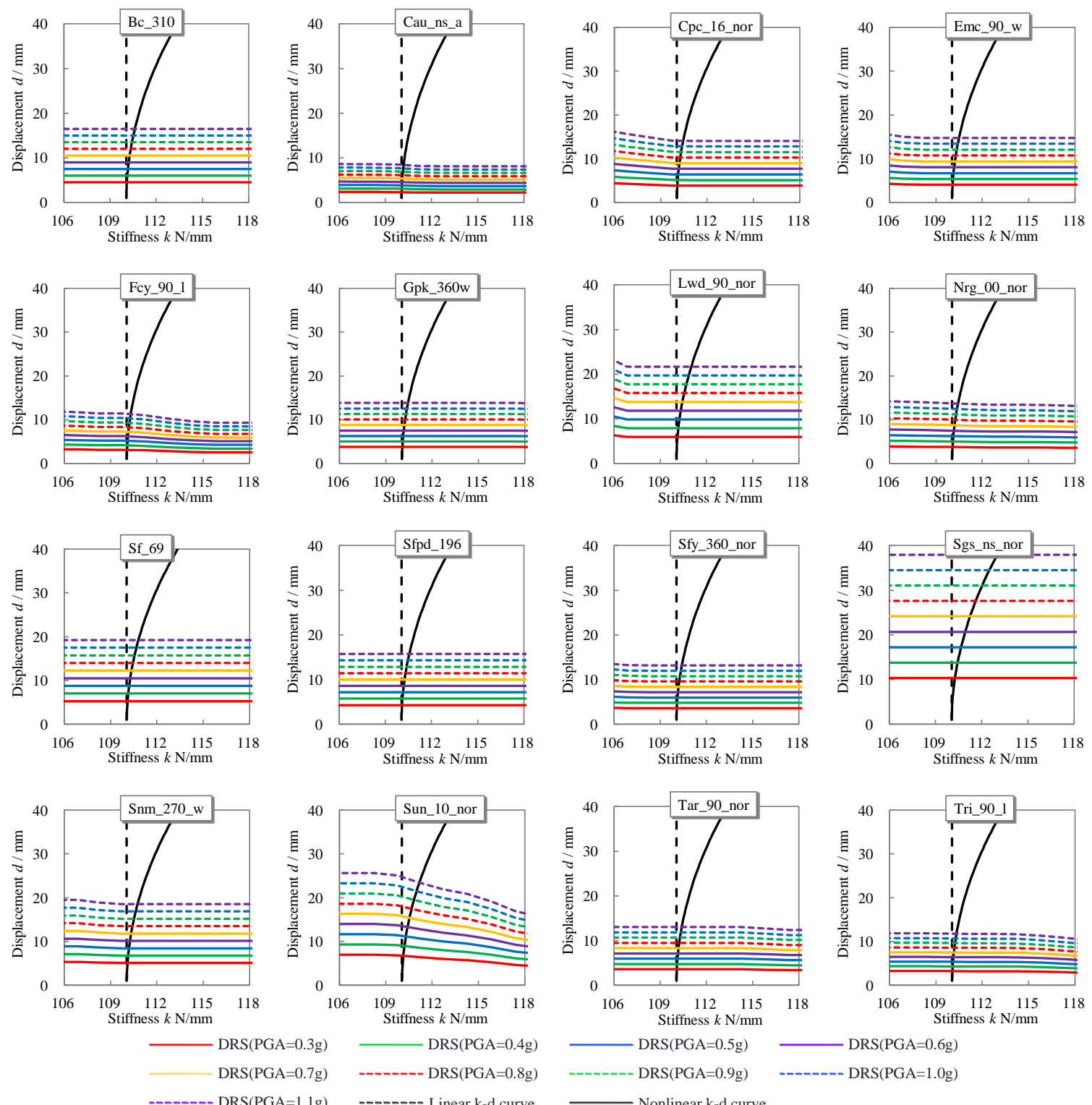

Fig.12. Graphic solutions for the ESDF responses of the $8^{\text {th }}$ mode

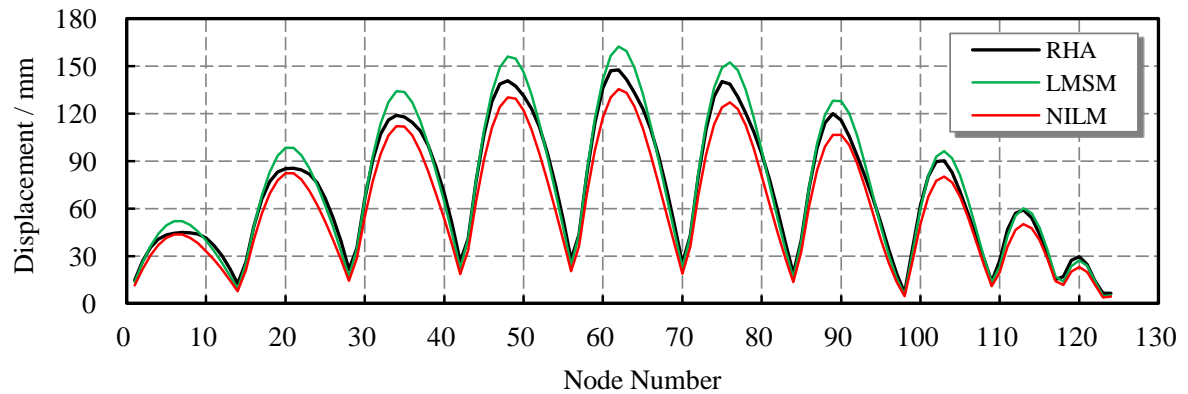

Fig.13. Displacement responses under the Bc_310 earthquake excitation with PGA=1.1g 


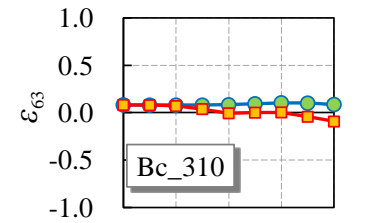

$\begin{array}{lllll}0.3 & 0.5 & 0.7 & 0.9 & 1.1\end{array}$ PGA / g
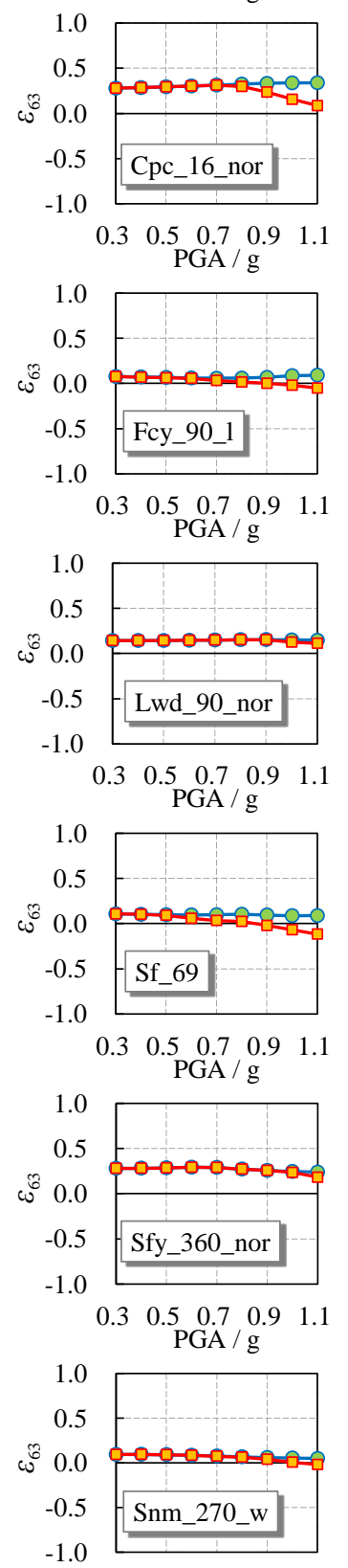

$\begin{array}{lllll}0.3 & 0.5 & 0.7 & 0.9 & 1.1\end{array}$ $\mathrm{PGA} / \mathrm{g}$

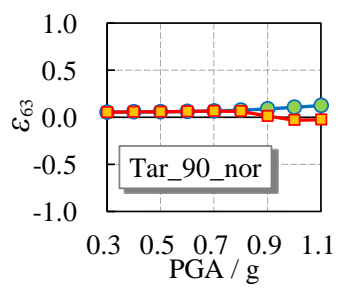

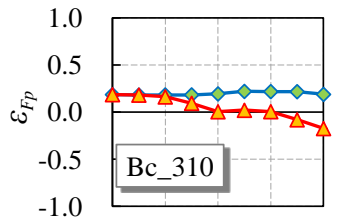

$\begin{array}{lllll}0.3 & 0.5 & 0.7 & 0.9 & 1.1\end{array}$

PGA / g
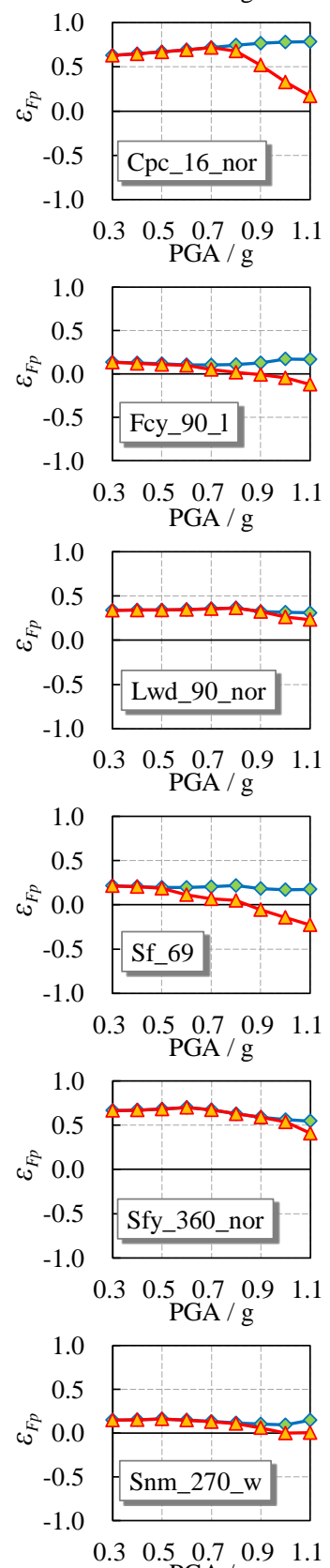

$\mathrm{PGA} / \mathrm{g}$

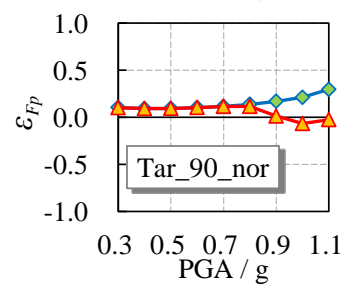

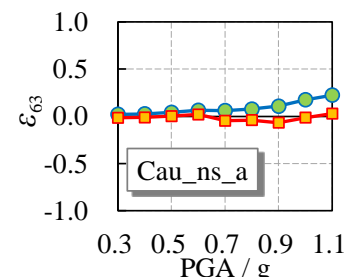
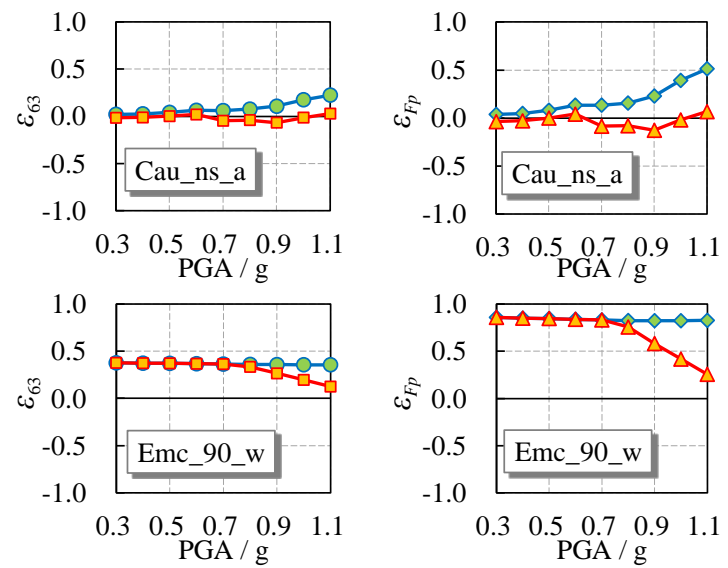

$\begin{array}{lllll}0.3 & 0.5 & 0.7 & 0.9 & 1.1\end{array}$ PGA / g
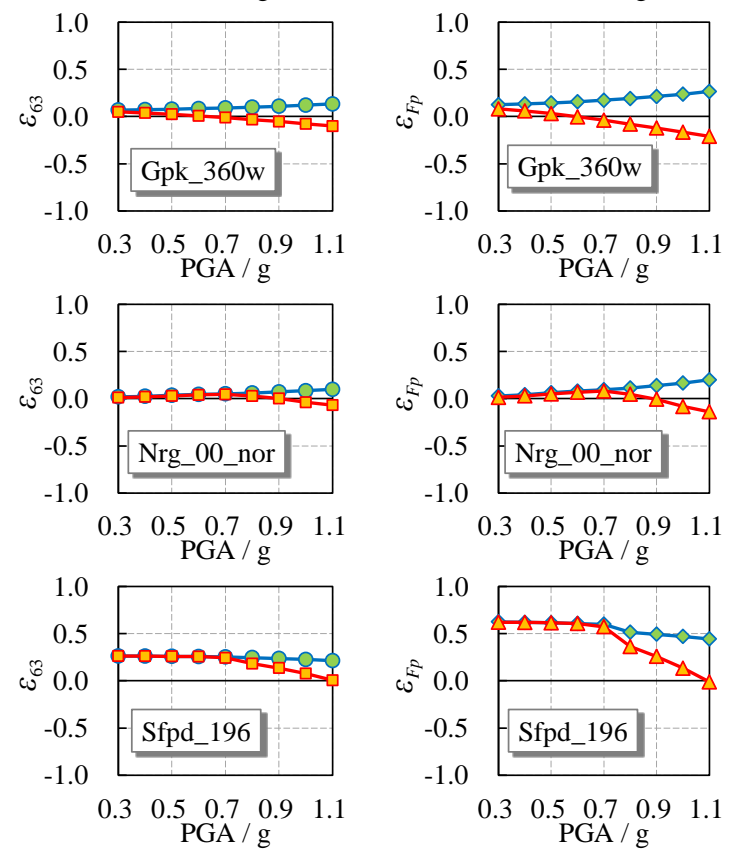

PGA / g
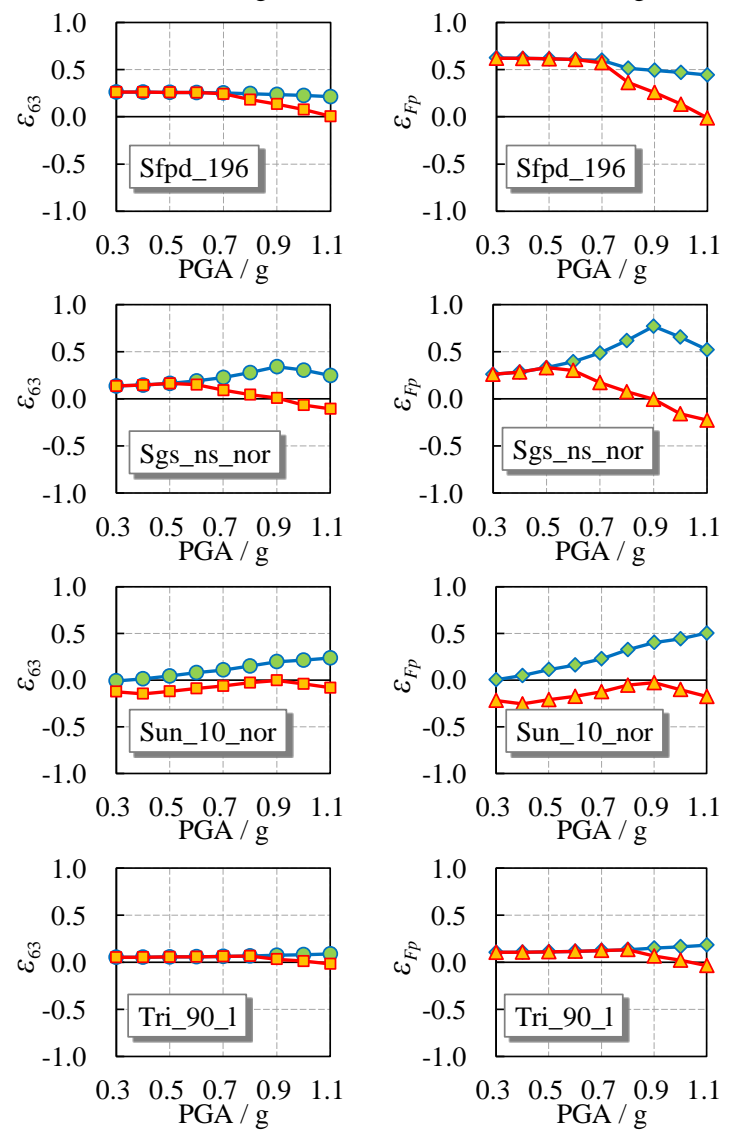

Fig.14. Values of $\varepsilon_{63}{ }^{\text {NILM }}, \varepsilon_{63}{ }^{\text {LMSM }}$ (a) and $\varepsilon_{F_{p}}{ }^{\text {NILM }}, \varepsilon_{F_{p}}{ }^{\text {LMSM }}$ (b) for the 16 seismic waves 